\title{
Optimal control in a multistage physiologically structured insect population model.
}

\author{
DELPHINE PICART* \\ Département des Mathématiques et Informatique, Université Antilles-Guyane, 97159 \\ Pointe-à-Pitre, France, \\ delphinepicart@hotmail.com \\ FABIO MILNER \\ Arizona State University, School of Mathematical and Statistical Sciences, Tempe, AZ \\ 85287-1804, United States, \\ milner@asu.edu
}

\begin{abstract}
We present an age- and stage-structured population model to study some methods of control of one of the most important grapevine pests, the European grapevine moth. We consider control by insecticides that reduce either the proportion of surviving eggs, larvae or both, as well as chemicals that cause mating disruption, thereby reducing the number of eggs laid. We formulate optimal control problems with cost functionals related to real-life costs in the wine industry, and we prove that these problems admit a unique solution. We also provide some numerical examples from simulation.
\end{abstract}

Keywords: population dynamics; age-structured population; partial differential equations; grape cultivar; hyperbolic systems; pest control; optimal control theory.

\section{Introduction.}

Crop industries are frequently confronted with pest populations that cause great damage and losses. Many such pests are insects as, for example, the boll weevil (Anthonomus grandis) in the cotton crop $^{13}$, the European grapevine moth (Lobesia botrana) in the wine industry ${ }^{8,18}$, or the apple leaf midge (Dasineura mali) in apple crops $^{6}$. The methods used to control these pest populations are mainly application of insecticides (e.g. Bt, growth regulator), mating disruption to a lesser degree ${ }^{19}$, and other modern methods currently in the testing process, such as biological control ${ }^{20}$ or development of transgenic plant mutants resistant to the particular insect ${ }^{13}$.

For many decades Lobesia botrana, the European grapevine moth (EGVM), has been a major concern in vineyards in Europe, North Africa, Asia and - very recently - also California ${ }^{5}$. In this paper, we model the first two control methods of this pest population with the goal of finding strategies that optimize the reduction of pest population size and amount of chemicals used.

* Corresponding author. 
Our model is built from the multistage physiologically structured population model presented in ${ }^{2,16}$, for any insect pest whose biological cycle consists of four main stages-egg, larva, female and male. The males are not considered here because the additional equation describing their population dynamics can be neglected in the mathematical analysis given in that paper without any loss of generality. Let $u^{e}, u^{l}$, and $u^{f}$ be, respectively, the age densities at time $t$ of the egg, larva and adult female moth populations. The dynamics of these populations in their free environment are modeled as in ${ }^{2,16}$ by the following so-called size-structured model in which the structure variable $a$ may represent size or another physiologically relevant variable:

$$
\left\{\begin{aligned}
\frac{\partial u^{e}}{\partial t}(t, a)+\frac{\partial}{\partial a}\left[g^{e}(E(t), a) u^{e}(t, a)\right]= & -\beta^{e}(E(t), a) u^{e}(t, a) \\
& -m^{e}(E(t), a) u^{e}(t, a), \quad(t, a) \in \Omega^{e}, \\
\frac{\partial u^{l}}{\partial t}(t, a)+\frac{\partial}{\partial a}\left[\left(g^{l}(E(t), a) u^{l}(t, a)\right]=\right. & -\beta^{l}(E(t), a) u^{l}(t, a) \\
& -m^{l}\left(P^{l}, E(t), a\right) u^{l}(t, a), \quad(t, a) \in \Omega^{l}, \\
\frac{\partial u^{f}}{\partial t}(t, a)+\frac{\partial}{\partial a}\left[\left(g^{f}(E(t), a) u^{f}(t, a)\right]=\right. & -m^{f}(E(t), a) u^{f}(t, a), \quad(t, a) \in \Omega^{f},
\end{aligned}\right.
$$

where $\Omega^{k}=[0, T] \times\left[0, L^{k}\right]$, for $k=e, l, f$, the boundary conditions, for $t \in[0, T]$, are given by

$$
\left\{\begin{array}{l}
g^{e}(E(t), 0) u^{e}(t, 0)=\int_{0}^{L^{f}} \beta^{f}\left(P^{f}, E(t), a\right) u^{f}(t, a) d a \\
g^{l}(E(t), 0) u^{l}(t, 0)=\int_{0}^{L^{e}} \beta^{e}(E(t), a) u^{e}(t, a) d a \\
g^{f}(E(t), 0) u^{f}(t, 0)=\int_{0}^{L^{l}} \beta^{l}(E(t), a) u^{l}(t, a) d a
\end{array}\right.
$$

and the initial conditions are

$$
u^{k}(0, a)=u_{0}^{k}(a), \quad a \in\left[0, L^{k}\right], \quad k=e, l, f .
$$

The total population for the $k$-stage is defined by

$$
P^{k}(t)=\int_{0}^{L^{k}} u^{k}(t, a) d a
$$

where $L^{k}$ is the maximum age for the stage, and $\mathrm{k}$ takes the value $e$ for egg, $l$ for larva and $f$ for female. The variable $E$ corresponds to the time-dependent vector $(T, H, R)$ modeling the changing climatic (Temperature and Humidity) and environmental (food Resource) conditions. The motivations leading to this mathematical framework of this population dynamics model and the explanation about the dependency of the variables $m^{l}$ and $\beta^{f}$ on the total population $P^{l}, P^{f}$ are given in the paper ${ }^{2}$. We just recall here to help the understanding of the next section that equations of system (1.2) describe all the key steps of the insect's biological cycle. In particular, the first equation models the birth dynamics, the second models the hatching dynamics and the third equation is a proxy for the adult flight dynamics. For $k=e, l, f$, the initial functions $u_{0}^{k}$ are assumed non-negative and integrable, the growth functions $g^{k}$-bounded in the age variable (with additional conditions 
specified in the appendix), and the k-stage mortality functions $m^{k}$-non-negative and locally bounded, satisfying the conditions

$$
\begin{aligned}
\lim _{a \rightarrow L^{k}} \int_{0}^{t} m^{k}\left(E(t), X^{k}(s ; t, a)\right) d s & =\infty, \quad a>X^{k}(t), \\
\lim _{a \rightarrow L^{k}} \int_{Z^{k}(0 ; t, a)}^{t} m^{k}\left(E(t), X^{k}(s ; t, a)\right) d s & =\infty, \quad a \leq X^{k}(t),
\end{aligned}
$$

for $t \in[0, T]$ and $k=e, f$, and the conditions

$$
\begin{aligned}
\lim _{a \rightarrow L^{l}} \int_{0}^{t} m^{l}\left(P^{l}, E(t), X^{l}(s ; t, a)\right) d s & =\infty, \quad a>X^{l}(t), \\
\lim _{a \rightarrow L^{l}} \int_{Z^{l}(0 ; t, a)}^{t} m^{l}\left(P^{l}, E(t), X^{l}(s ; t, a)\right) d s & =\infty, \quad a \leq X^{l}(t),
\end{aligned}
$$

in order to ensure that all individuals from the first two life stages transition into the next stage by the maximal age for their stage and that all female adult moths die by the maximal age for the species.

The functions $X^{k}$ and $Z^{k}$ (for $k=e, l, f$ ) parametrize, respectively with time $t$ and age $a$ as parameters, the characteristic curves of (1.1) defined by

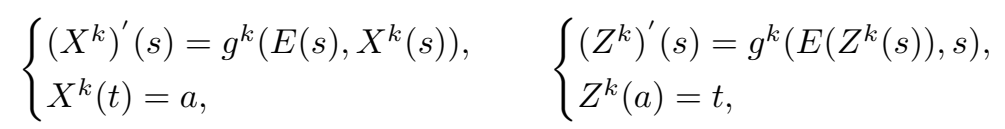

and they are assumed to be Lipschitz continuous in the first variable, with Lipschitz constant $m_{K}$. The function $\beta^{e}$ models the transition between the egg and larval stages, whereas the function $\beta^{l}$ models the transition between the larval and the adult female moth stages. The function $\beta^{f}$ represents the age-specific fertility. These last three functions are non-negative and bounded, and they satisfy

$$
0 \leq \underline{\beta}^{k} \leq \beta^{k}(E(t), a), \beta^{f}\left(P^{f}, E(t), a\right) \leq \bar{\beta}^{k}, \quad k=e, l .
$$

Moreover, the fertility function is assumed Lipschitz continuous in the first variable, $P^{f}$, with Lipschitz constant $\beta_{K}$.

In the next section we motivate and describe the control problems we shall analyze in this paper. In Section 3, the existence of a solution for the resulting optimal control problem is proved whereas in Section 4 the uniqueness of the optimal control pair is obtained through the use of Ekeland's principle ${ }^{3,7}$. Section 5 is devoted to the numerical characterization of the optimal control through simulations. Finally, in Section 6, we summarize our results and draw some conclusions.

\section{New models for control.}

We begin by describing how the two types of control we consider in this paper can be built into the general model (1.1)-(1.3). Then, we describe the cost function we consider and we give a rationale for the form of its functional dependence on the 
control(s) and densities.

Insecticides cause a direct decline in egg and larval populations by targeting either population or both. For example, egg pesticides (ovicides) are sprayed just before the onset of the egg laying dynamics in order to kill a maximal number of eggs, thus guiding our model to include a modification of (1.2) as follows. We let $v_{1}$ be an indicator of the egg pesticide application at time $t$ and age $a$, and we let $\alpha_{1} \in[0,1]$ represent its efficiency. More specifically, $\alpha_{1}$ is the percentage of eggs killed by the ovicide per unit time, assumed to be the same for eggs of all ages. As for the control, $v_{1}$, we shall restrict it to take values in $[0,1]$, with 0 indicating it should not be applied at the particular time and values in $(0,1]$ should be interpreted as the dilution to use for optimal cost, which we shall assume results in a linear decrease of its efficiency. In the linear case - when there is no density dependence in the fertility function - our simulations seem to indicate that the optimal control actually takes only the values 0 and 1 , thus working just as a switch that turns on and off the application of pesticide.

The pest population dynamics under the effect of egg pesticide control is modeled by (1.1)-(1.3), except that the boundary condition for the egg density is now given by

$$
g^{e}(E(t), 0) u^{e}(t, 0)=\int_{0}^{L^{f}}\left[1-\alpha_{1} v_{1}(t, a)\right] \beta^{f}\left(P^{f}, E(t), a\right) u^{f}(t, a) d a .
$$

The number of eggs surviving the control is now computed from the number of eggs laid without the use of control by subtracting the number of eggs killed by the egg-pesticide.

Larval pesticides are applied during the "black head" stage of egg development. We let $v_{2}$ be the indicator of larval pesticide application at time $t$ and age $a$, and we let $\alpha_{2} \in[0,1]$ represent its efficiency. The pest population dynamics under the effect of larval pesticide control is modeled by (1.1)-(1.3) with the equation describing the egg population dynamics modified to

$$
\begin{aligned}
\frac{\partial u^{e}}{\partial t}(t, a)+\frac{\partial}{\partial a}\left[g^{e}(E(t), a) u^{e}(t, a)\right] & =-\beta^{e}(E(t), a) u^{e}(t, a) \\
& -\left[m^{e}(E(t), a)+\alpha_{2} v_{2}(t, a)\right] u^{e}(t, a), \quad(t, a) \in \Omega^{e} .
\end{aligned}
$$

The mating disruption consists of disturbing mating by diffusing the female sex pheromone from dispensers thereby reducing the mating rate and, consequently, the number of eggs laid; see ${ }^{19}$ for a more complete description of the mechanism. The pheromone dispensers must be applied before the beginning of mating, typically during the egg laying dynamics. Therefore, this method of control can be modeled in exactly the same way as control by ovicide, and that is the approach we shall take in this paper. 
In summary, the population dynamics under these pest population control methods is described by

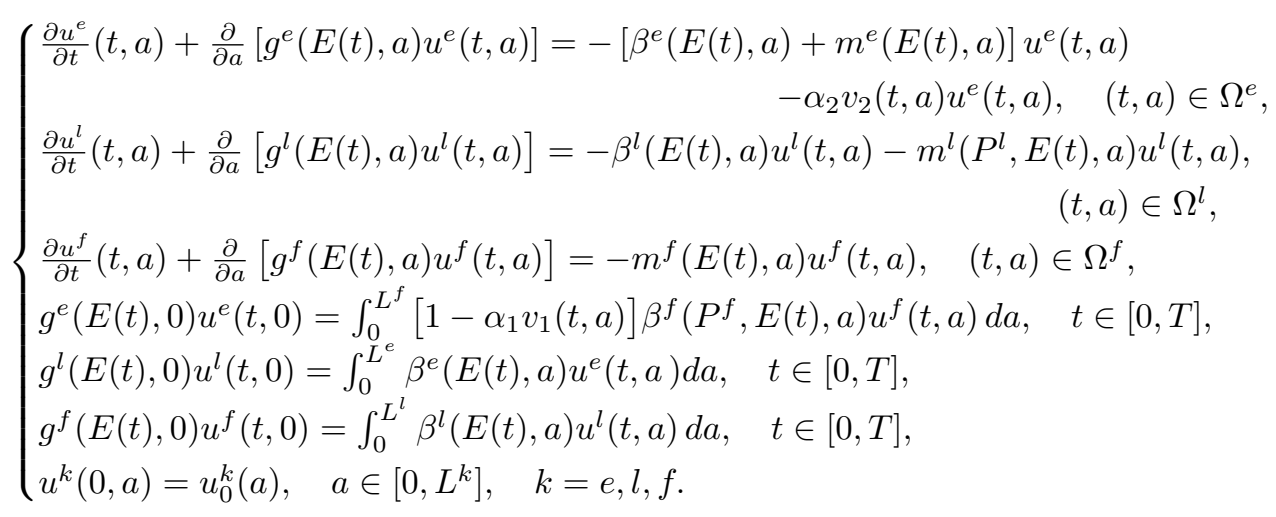

We remark that for both pesticides we have assumed here that the control product targets a specific population that is function of the real time of the product application: ovicide only affects the newly laid eggs and not the aged eggs, larvicide kills at the egg stage and not at the larval. If we assume rather that the applied product kills any eggs or any larvae, whatever their developmental stage, then system (2.3) should be modified by adding the following 3 terms:

i) $-\alpha_{1} v_{1}(t, a) u^{e}(t, a)$ in the equation related to the egg population dynamics to model the eggs killed by the ovicide,

ii) $-\alpha_{2} v_{2}(t, a) u^{l}(t, a)$ in the equation related to the larval population dynamics to model the larvae killed by the larvicide,

iii) and the boundary equation of the larval stage is written by

$$
g^{l}(E(t), 0) u^{l}(t, 0)=\int_{0}^{L^{e}}\left[1-\alpha_{2} v_{2}(t, a)\right] \beta^{e}(E(t), a) u^{e}(t, a) d a,
$$

to model the number of new larvae (i.e. newly hatched eggs) killed as a consequence of the larvicide application.

The vector function $v=\left(v_{1}, v_{2}\right)$ represents the controls, which take values in the set $[0,1]$ thus maintaining the positivity of the density functions in $(2.3)$. The mathematical analysis of this system is quite similar to this one developed in ${ }^{2}$, leading to the existence and uniqueness of solutions $u^{k}$, for $k=e, l, f$, of (2.3) and to the following a priori estimates that we shall state without proof: for $k=e, l, f$ and $t \in[0, T]$,

$$
\left\|u^{k}(t, \cdot)\right\|_{L^{1}\left(\left[0, L^{k}\right]\right)} \leq \bar{\beta}^{f} T P(0) e^{\bar{\beta}^{f} T}=C\left(\bar{\beta}^{f}, T\right) P(0),
$$

where $P(0)$ is the sum of all initial populations.

Next we establish another technical result, namely the Lipschitz-dependence of the densities on the controls. More precisely, we have the following result. 
Theorem 1. Let $v=\left(v_{1}, v_{2}\right)$ and $\tilde{v}=\left(\tilde{v}_{1}, \tilde{v}_{2}\right)$ represent two controls. Then, there exist positive constants $D^{k}, k=e, l, f$, such that

$$
\left\|u_{v}^{k}(t, \cdot)-u_{\tilde{v}}^{k}(t, \cdot)\right\|_{L^{1}\left(\Omega^{k}\right)} \leq D^{k}\|v-\tilde{v}\|_{L^{\infty}},
$$

for any $t \in[0, T]$, where $u_{v}^{k}$ and $u_{\tilde{v}}^{k}$ are, respectively, the solutions of (2.3) with the controls $v$ and $\tilde{v}$.

Proof: See the Appendix.

Let now $\mu$ represent the financial cost of larval damage per larva per unit time, and let $\eta_{1} / L^{f}$ and $\eta_{2} / L^{e}$ represent, respectively, the cost per unit time of carrying out an application of ovicide (and/or mating disruption pheromones) and of larvicide. We are then naturally led to study the following optimal control problem:

$$
[\mathrm{P}]: \text { Find } \min _{\left(v_{1}, v_{2}\right) \in K} \mathcal{J}\left(v_{1}, v_{2}\right) \text {, }
$$

where

$\mathcal{J}\left(v_{1}, v_{2}\right)=\left[\mu \int_{\Omega^{l}} u^{l}(t, a) d a d t+\frac{\eta_{1}}{L^{f}} \int_{\Omega^{f}}\left(v_{1}\right)^{2}(t, a) d a d t+\frac{\eta_{2}}{L^{e}} \int_{\Omega^{e}}\left(v_{2}\right)^{2}(t, a) d a d t\right]$,

and $u^{l}$ is the solution of (2.3) and, $K$ is the convex compact set given by

$$
K=\left\{v \in L^{\infty}\left(\Omega^{f} \times \Omega^{e}\right): v_{1}: \Omega^{f} \rightarrow[0,1], v_{2}: \Omega^{e} \rightarrow[0,1]\right\} .
$$

Note that the integrals forming the cost functional represent, respectively, the total financial cost of grape losses due to the infestation, and the total cost of the applications of ovicides (and/or mating disruption hormones), and of larvicides. Since one does not know the relation between the cost of an intervention and the amount of product used the quadratic form on the control functions may be appropriate.

In general the control application is equidistributed among eggs/larvae/insects of all ages so that $v_{1}$ and $v_{2}$ are age-independent. In such case we can simplify the form of the functional to

$$
\mathcal{J}\left(v_{1}, v_{2}\right)=\left[\mu \int_{\Omega^{l}} u^{l}(t, a) d a d t+\eta_{1} \int_{0}^{T}\left(v_{1}\right)^{2}(t) d t+\eta_{2} \int_{0}^{T}\left(v_{2}\right)^{2}(t) d t\right] .
$$

We can find in the literature several results about optimal control for problems governed by parabolic equations ${ }^{1,12,21}$, by integro-difference equations ${ }^{11}$ and, just like here, by hyperbolic equations ${ }^{3,4,9,10}$. In all these articles, however, the cost functional is expressed as a nonlinear (quadratic) function of the control(s) and the density, whereas this dependency is linear in our problem $[P]$. Barbu and Iannelli ${ }^{3}$ determined the optimal control to reduce the growth of a population. They modified the Gurtin-Mac Camy model to include control functions as factors of the vital rates. Fister and Lenhart ${ }^{9,10}$ and Busoni and Matuccia ${ }^{4}$ studied the optimal proportion of harvested individuals in two populations. The controls were defined in this case for the age-structured population equations as a functions that could be age- and time-dependent. 


\section{Existence of a solution.}

In this section we establish the existence of a solution for problem $[\mathrm{P}]$. This is quite straightforward in contrast with the proof of uniqueness that we leave for the next section as it requires considerably more work.

Theorem 2. The optimal control problem $[P]$ admits a solution $\left(v_{1}, v_{2}\right)$.

Proof: Let $d=\min _{\left(v_{1}, v_{2}\right) \in K} \mathcal{J}\left(v_{1}, v_{2}\right)$ and let $\left\{v_{1}^{n}, v_{2}^{n}\right\}_{n \in \mathbb{N}}$ be a minimizing sequence such that

$$
d<\mathcal{J}\left(v_{1}^{n}, v_{2}^{n}\right) \leq d+\frac{1}{n} .
$$

Since the sequence $\left\{v_{1}^{n}, v_{2}^{n}\right\}_{n \in \mathbb{N}}$ belongs to the space $\mathrm{K}$, there exists a convergent subsequence $\left\{v_{1}^{n_{k}}, v_{2}^{n_{k}}\right\}_{k \in \mathbb{N}}$ in $L^{2}\left(\Omega^{f}\right) \times L^{2}\left(\Omega^{e}\right)$; let $\left(v_{1}^{*}, v_{2}^{*}\right)$ be its limit. Consider now the sequence of density functions $\left\{\left[u^{e}\right]^{n_{k}}\right\}_{k \in \mathbb{N}}$ defined by (2.3), given along the characteristic curves by

$$
\left[u^{e}\right]^{n_{k}}(t, a)=\left\{\begin{array}{l}
{\left[u_{0}^{e}\right]^{n_{k}}\left(X^{e}(0 ; t, a)\right) e^{-\int_{0}^{t}\left[h^{e}\right]^{n_{k}}\left(s, X^{e}(s ; t, a)\right) d s}, \quad a>X^{e}(t),} \\
\frac{\left[u^{e}\right]^{n_{k}}\left(Z^{e}(0 ; t, a), 0\right)}{g^{e}\left(E\left(Z^{e}(0 ; t, a)\right), 0\right)} e^{-\int_{Z^{e}(0 ; t, a)}^{t}\left[h^{e}\right]^{n_{k}}\left(s, X^{e}(s ; t, a)\right) d s}, \quad a \leq X^{e}(t),
\end{array}\right.
$$

where

$\left\{\begin{array}{l}{\left[h^{e}\right]^{n_{k}}(t, a)=\beta^{e}(E(t), a)+m^{e}(E(t), a)+\alpha_{2} v_{2}^{n_{k}}(t, a)+\partial_{a}\left[g^{e}\right]^{n_{k}}(E(t), a),} \\ {\left[g^{e}\right]^{n_{k}}(E(t), 0)\left[u^{e}\right]^{n_{k}}(t, 0)=\int_{0}^{L^{f}}\left[1-\alpha_{1} v_{1}^{n_{k}}(t, a)\right] \beta^{f}\left(\left[P^{f}\right]^{n_{k}}, E(t), a\right)\left[u^{f}\right]^{n_{k}}(t, a) d a .}\end{array}\right.$

These relations show explicitly the dependence of the densities on the sequence $\left\{v_{1}^{n_{k}}, v_{2}^{n_{k}}\right\}_{k \in \mathbb{N}^{*}}$. Similarly, we can see the dependence of the density functions $\left\{\left[u^{l}\right]^{n_{k}}\right\}_{k \in \mathbb{N}}$ and $\left\{\left[u^{f}\right]^{n_{k}}\right\}_{k \in \mathbb{N}}$ defined by (2.3) on $\left\{v_{1}^{n_{k}}, v_{2}^{n_{k}}\right\}_{k \in \mathbb{N}^{\prime}}$. From the hypothesis made in Section 2 we can easily prove (as described in ${ }^{14}$ ) that the sequence of functions $\left[P^{l}\right]^{n_{k}}(t)=\int_{0}^{L^{l}}\left[u^{l}\right]^{n_{k}}(t, a) d a, k \in \mathbb{N}$, converges strongly to $\left[P^{l}\right]^{*}(t)=\int_{0}^{L^{l}}\left[u^{l}\right]^{*}(t, a) d a$, with $\left[u^{l}\right]^{*}$ solution of $(2.3)$ with $\left(v_{1}^{*}, v_{2}^{*}\right)$, since the sequence is bounded in $H^{!}$. Finally, we see that $\lim _{n \rightarrow \infty} \mathcal{J}\left(v_{1}^{n}, v_{2}^{n}\right)=\mathcal{J}\left(v_{1}^{*}, v_{2}^{*}\right)$ and, from the first inequality of $(3.1)$, it follows that $\left(v_{1}^{*}, v_{2}^{*}\right)$ is a solution of $[P]$.

\section{Uniqueness of the solution.}

The method we shall use to establish uniqueness is based on that of Barbu and Iannelli's paper ${ }^{3}$. We carry out the same procedure they used and utilize their notation. 
We shall need the following dual problem associated to (2.3),

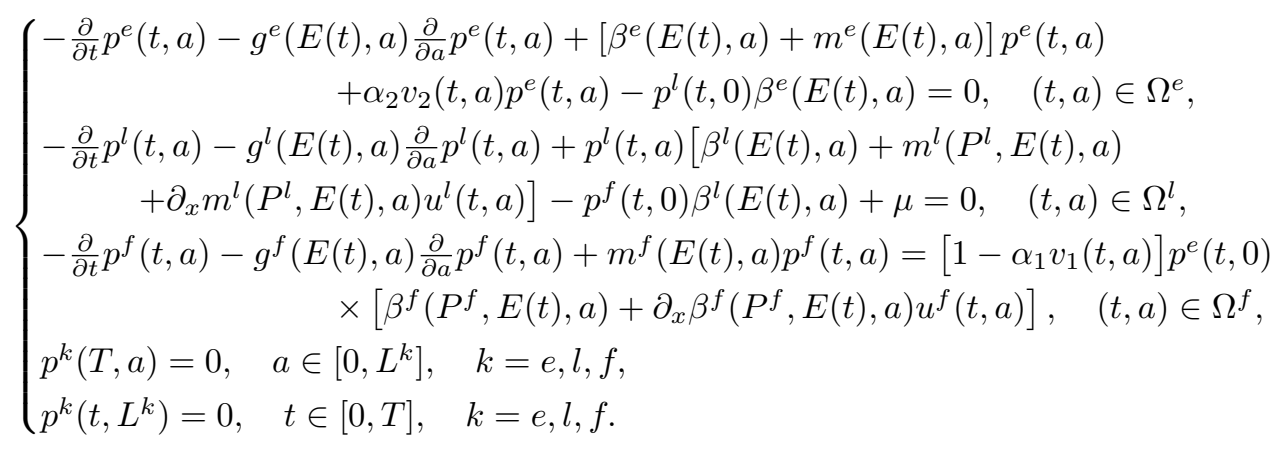

Using the method of characteristics, we can produce an explicit solution for this system that satisfies the Lipschitz conditions given in theorem 3 below that will be useful later.

Theorem 3. The system (4.1) admits a unique solution and, for $t \in[0, T]$, it satisfies

$$
\begin{aligned}
& \left|p_{v}^{e}(., 0)-p_{\tilde{v}}^{e}(., 0)\right|_{\infty} \leq G\|v-\tilde{v}\|_{L^{\infty}}, \\
& \left\|p_{v}^{e}-p_{\tilde{v}}^{e}\right\|_{L^{2}\left(\Omega^{e}\right)} \leq H\|v-\tilde{v}\|_{L^{\infty}},
\end{aligned}
$$

where $G$ and $H$ are positive constants, $p_{v}^{e}$ and $p_{\tilde{v}}^{e}$ are solutions of (4.1) corresponding, respectively, to the controls $v$ and $\tilde{v}$, and $|\cdot|_{\infty}$ denotes the $L^{\infty}([0, T])$-norm.

Proof: See the Appendix.

The dual problem associated with $[P]$ is given by (4.1) and its optimality conditions are given in the following lemma.

Lemma 1. The optimality conditions for problem $[P]$ are

$$
\begin{aligned}
& 2 \eta_{1} v_{1}(\cdot, a)+\alpha_{1} p^{e}(\cdot, 0) \beta^{f}\left(P^{f}, \cdot, a\right) u^{f}(\cdot, a)=0, \quad a \in\left[0, L^{f}\right], \\
& 2 \eta_{2} v_{2}(\cdot, a)+\alpha_{2} u^{e}(\cdot, a) p^{e}(\cdot, a)=0, \quad a \in\left[0, L^{e}\right],
\end{aligned}
$$

identically in $[0, T]$ where $v=\left(v_{1}, v_{2}\right)$ is the optimal control, $\left(u^{f}, u^{e}\right)$ satisfy (2.3) for the optimal $v$, and $p^{e}$ is the solution of (4.1) satisfying the condition

$$
-\frac{2 \eta_{1}}{\underline{\beta}^{f} P_{\min }^{f}} \leq p^{e}(t, 0) \leq 0
$$

where $P_{\text {min }}^{f}>0$ is the minimum of the adult female population size for $t \in[0, T]$.

Proof: Follows directly from computing the derivatives of the cost functional with respect to the controls. 
We then prove the uniqueness of solutions to problem $[P]$. Following Barbu and Iannelli ${ }^{3}$ we define the function

$$
\Phi(v)= \begin{cases}\mathcal{J}^{\prime}(v), & v \in K, \\ +\infty, & \text { otherwise },\end{cases}
$$

so that, for $\epsilon \geq 0$, there exists $v_{\epsilon}=\left(v_{\epsilon}^{1}, v_{\epsilon}^{2}\right) \in K$ satisfying

$$
\begin{aligned}
& \Phi\left(v_{\epsilon}\right) \leq \inf _{v \in K} \Phi(v)+\epsilon, \\
& \Phi\left(v_{\epsilon}\right) \leq \inf _{v \in K}\left(\Phi(v)+\sqrt{\epsilon} \sum_{i=1}^{2}\left\|v_{i}-v_{\epsilon}^{i}\right\|_{L^{1}}\right) .
\end{aligned}
$$

Computing the derivatives with respect to the vector $v$ of the Lagrangian of the cost functional defined by the expression in parentheses in (4.5) we get the following relations,

$$
\left\{\begin{array}{r}
\int_{\Omega^{f}}\left[2 \eta_{1} v_{\epsilon}^{1}(t, a)+\alpha_{1} p^{e}(t, 0) \beta^{f}\left(P^{f}, E(t), a\right) u^{f}(t, a)\right] h_{1}(t, a) d a d t \\
\quad+\sqrt{\epsilon} \int_{\Omega^{f}} h_{1}(t, a) d a d t \geq 0, \\
\int_{\Omega^{e}}\left[2 \eta_{2} v_{\epsilon}^{2}(t, a)+\alpha_{2} u^{e}(t, a) p^{e}(t, a)\right] h_{2}(t, a) d a d t+\sqrt{\epsilon} \int_{\Omega^{e}} h_{2}(t, a) d a d t \geq 0,
\end{array}\right.
$$

for all $h=\left(h_{1}, h_{2}\right) \in T_{v_{\epsilon}}(K)$, where the set $T_{v_{\epsilon}}(K)$ represents the tangent cone to $K$ at $v_{\epsilon}$. The two expressions in the square brackets are the components of a vector in $L^{1}\left(\Omega^{f} \times \Omega^{e}\right)$ and then, as a consequence of proposition 5.3 of Barbu and Iannelli ${ }^{3}$, we have the existence of a function $\theta_{\epsilon}=\left(\theta_{\epsilon}^{1}, \theta_{\epsilon}^{2}\right) \in L^{\infty}\left(\Omega^{f} \times \Omega^{e}\right),\left|\theta_{\epsilon}^{i}\right|<1$ for $i=1,2$, such that the functions

$$
\left\{\begin{array}{l}
2 \eta_{1} v_{\epsilon}^{1}(t, a)+\alpha_{1} p^{e}(t, 0) \beta^{f}\left(P^{f}, E(t), a\right) u^{f}(t, a)+\sqrt{\epsilon} \theta_{\epsilon}^{1}, \quad(t, a) \in \Omega^{f}, \\
2 \eta_{2} v_{\epsilon}^{2}(t, a)+\alpha_{2} u^{e}(t, a) p^{e}(t, a)+\sqrt{\epsilon} \theta_{\epsilon}^{2}, \quad(t, a) \in \Omega^{e},
\end{array}\right.
$$

are the components of a vector in $N_{v_{\epsilon}}(K)$, the normal cone of $K$ at $v_{\epsilon}$. We can deduce from the above that the solution $v_{\epsilon}$ is given by

$$
\left\{\begin{array}{l}
v_{\epsilon}^{1}(t, a)=\mathcal{L}\left(-\frac{\alpha_{1}}{2 \eta_{1}} p^{e}(t, 0) \beta^{f}\left(P^{f}, E(t), a\right) u^{f}(t, a)-\frac{\sqrt{\epsilon}}{2 \eta_{1}} \theta_{\epsilon}^{1}(t, a)\right), \quad(t, a) \in \Omega^{f}, \\
v_{\epsilon}^{2}(t, a)=\mathcal{L}\left(-\frac{\alpha_{2}}{2 \eta_{2}} u^{e}(t, a) p^{e}(t, a) d s-\frac{\sqrt{\epsilon}}{2 \eta_{2}} \theta_{\epsilon}^{2}(t, a)\right), \quad(t, a) \in \Omega^{e}
\end{array}\right.
$$

where the mapping $\mathcal{L}$ is given by

$$
(\mathcal{L} f)(t, a)=\left\{\begin{array}{l}
f(t, a), \quad \underline{g} \leq f(t, a) \leq \bar{g} \\
\underline{g}, \quad f(t, a) \leq \underline{g} \\
\bar{g}, \quad f(t, a) \geq \bar{g}
\end{array}\right.
$$

We can now prove the following uniqueness result. 
Theorem 4. Let us define the following positive conditions,

$$
\left\{\begin{array}{l}
\gamma_{1}=\bar{\beta}^{f}\left\|u^{f}\right\|_{L^{\infty}} G+\bar{\beta}^{f}\left|p^{e}(\cdot, 0)\right|_{\infty} D^{f}+\beta_{K}\left|p^{e}(\cdot, 0)\right|_{\infty}\left\|u^{f}\right\|_{L^{\infty}} D^{f} \\
\gamma_{2}=\left\|p^{e}\right\|_{L^{\infty}} D^{e}+(L T)^{1 / 2}\left\|u^{e}\right\|_{L^{\infty}} H
\end{array}\right.
$$

where $D^{f}$ and $D^{e}$ are positive constants given in Theorem 1 whereas $G$ and $H$ are those given in Theorem 3. If, $0 \leq \frac{\gamma_{1}}{2 \eta_{1}}, \frac{\gamma_{2}}{2 \eta_{2}}<1$, then problem $[P]$ has a unique solution $v=\left(v_{1}, v_{2}\right) \in K$.

The above conditions $\left(\gamma_{1}, \gamma_{2}\right)$ guaranteeing the uniqueness are dependent on the density functions $u^{e}$ and $u^{f}$ that are solutions of the system (2.3) and linked with the dual variables $p^{e}$ and, independent on the control functions $\left(v_{1}, v_{2}\right)$. Since all terms of these conditions are positive then we can simplify them as follow

$$
\bar{\beta}^{f}\left\|u^{f}\right\|_{L^{\infty}} G<2 \eta_{1}, \quad \text { and } \quad(L T)^{1 / 2}\left\|u^{e}\right\|_{L^{\infty}} G<2 \eta_{2},
$$

because $H=G$ (see Annex) and deduce this condition on the maximal value on the female density functions,

$$
\left\|u^{f}\right\|_{L^{\infty}} \leq \frac{\eta_{1}}{\eta_{2}} \frac{(L T)^{1 / 2}}{\underline{g}^{e}} P_{\max }^{f}
$$

that requires to be lesser than the maximal number of female $P_{\max }^{f}$ times a positive constant.

Proof: Let $\mathcal{E}$ be the Banach space $\left(L^{\infty}\left(\Omega^{f} \times \Omega^{e}\right)\right)$, and consider the mapping $(\mathcal{F} v)(t): \mathcal{E} \rightarrow \mathcal{E}$ given by

$$
\left\{\begin{array}{l}
\left(\mathcal{F}_{1} v\right)(t, a)=\mathcal{L}\left(-\frac{\alpha_{1}}{2 \eta_{1}} p_{v}^{e}(t, 0) \beta^{f}\left(P_{v}^{f}, E(t), a\right) u_{v}^{f}(t, a)\right), \\
\left(\mathcal{F}_{2} v\right)(t, a)=\mathcal{L}\left(-\frac{\alpha_{2}}{2 \eta_{2}} u_{v}^{e}(t, a) p_{v}^{e}(t, a)\right)
\end{array}\right.
$$

where $\left(p_{\tilde{v}}^{e}, u_{\tilde{v}}^{e}, u_{\tilde{v}}^{f}\right)$ and $\left(p_{v}^{e}, u_{v}^{e}, u_{v}^{f}\right)$ are solutions of (4.1) and (2.3) with the controls $\tilde{v}$ and $v$, respectively. First, we prove that the mapping $\mathcal{F} v$ is a contraction. Let $v=\left(v_{1}, v_{2}\right)$ and $\tilde{v}=\left(\tilde{v}_{1}, \tilde{v}_{2}\right)$ be two controls; then, from (4.6) we deduce that

$$
\begin{aligned}
\left|\mathcal{F}_{1} v(t, a)-\mathcal{F}_{1} \tilde{v}(t, a)\right| \leq & \frac{1}{2 \eta_{1}}\left|p_{\tilde{v}}^{e}(t, 0)-p_{v}^{e}(t, 0)\right|\left|\beta^{f}\left(P_{v}^{f}, E(t), a\right) u_{\tilde{v}}^{f}(t, a)\right| \\
& +\frac{1}{2 \eta_{1}}\left|p_{v}^{e}(t, 0)\right|\left|\beta^{f}\left(P_{v}^{f}, E(t), a\right)-\beta^{f}\left(P_{\tilde{v}}^{f}, E(t), a\right)\right|\left|u_{\tilde{v}}^{f}(t, a)\right| \\
& +\frac{\bar{\beta}^{f}}{2 \eta_{1}}\left|p_{v}^{e}(t, 0)\right|\left|u_{\tilde{v}}^{f}(t, a)-u_{v}^{f}(t, a)\right|, \\
\left|\mathcal{F}_{2} v(t, a)-\mathcal{F}_{2} \tilde{v}(t, a)\right| \leq & \frac{1}{2 \eta_{2}}\left|u_{\tilde{v}}^{e}(t, s)-u_{v}^{e}(t, s)\right|\left|p_{v}^{e}(t, s)\right| \\
& +\frac{1}{2 \eta_{2}} u_{\tilde{v}}^{e}(t, s)\left|p_{\tilde{v}}^{e}(t, s)-p_{v}^{e}(t, s)\right| .
\end{aligned}
$$


Using theorems 1 and 3, the above inequalities become

$$
\begin{aligned}
\left|\mathcal{F}_{1} v(t, a)-\mathcal{F}_{1} \tilde{v}(t, a)\right| \leq & \frac{1}{2 \eta_{1}}\left[\bar{\beta}^{f}\left\|u_{\tilde{v}}^{f}\right\|_{L^{\infty}} G+\bar{\beta}^{f}\left|p_{v}^{e}(., 0)\right|_{\infty} D^{f}\right. \\
& \left.+\beta_{K}\left|p_{v}^{e}(., 0)\right|_{\infty}\left\|u_{\tilde{v}}^{f}\right\|_{L^{\infty}} D^{f}\right]\|\tilde{v}-v\|_{L^{\infty}}, \\
\left|\mathcal{F}_{2} v(t, a)-\mathcal{F}_{2} \tilde{v}(t, a)\right| \leq & \frac{1}{2 \eta_{2}}\left[\left\|p_{v}^{e}\right\|_{L^{\infty}} D^{e}+(L T)^{1 / 2}\left\|u_{\tilde{v}}^{e}\right\|_{L^{\infty}} H\right]\|\tilde{v}-v\|_{L^{\infty}},
\end{aligned}
$$

where the estimate of the $L^{2}$-norm of the dual variable $p^{e}$ is given in the Appendix. Let us denote by $\gamma_{1}$ and $\gamma_{2}$, respectively, the terms inside the square brackets of the first and second inequalities above, so that those inequalities are now rewritten as

$$
\left\{\begin{array}{l}
\left|\mathcal{F}_{1} v(t, a)-\mathcal{F}_{1} \tilde{v}(t, a)\right| \leq \frac{\gamma_{1}}{2 \eta_{1}}\|\tilde{v}-v\|_{L^{\infty}}, \\
\left|\mathcal{F}_{2} v(t, a)-\mathcal{F}_{2} \tilde{v}(t, a)\right| \leq \frac{\gamma_{2}}{2 \eta_{2}}\|\tilde{v}-v\|_{L^{\infty}}
\end{array}\right.
$$

Since we have

$$
0 \leq \frac{\gamma_{1}}{2 \eta_{1}}, \frac{\gamma_{2}}{2 \eta_{2}}<1
$$

the mapping $\mathcal{F}$ is a contraction admitting a unique fixed point, say $v^{*}$. Next, we prove that the solution of $\left[P^{\prime}\right]$ is unique, and it is $v^{*}$. Indeed, for $i=1,2$, we have

$$
\left\|v_{i}^{*}-v_{\epsilon}^{i}\right\|_{L^{\infty}} \leq\left|\mathcal{F}_{i}\left(v_{i}^{*}\right)-\mathcal{F}_{1}\left(v_{\epsilon}^{i}\right)\right|+\frac{\sqrt{\epsilon}}{2 \eta_{i}}\left|\theta_{\epsilon}^{i}(t, a)\right|,
$$

which implies, using (4.7), that for $i=1,2$,

$$
\left\|v_{i}^{*}-v_{\epsilon}^{i}\right\|_{L^{\infty}} \leq\left[1-\frac{\gamma_{i}}{2 \eta_{i}}\right]^{-1} \frac{\sqrt{\epsilon}}{2 \eta_{i}}
$$

Finally, we conclude that $\lim _{\epsilon \rightarrow 0} v_{\epsilon}=v^{*}$ and, by inequality (4.4), $v^{*}$ is the unique minimizer of $\left[P^{\prime}\right]$ and, therefore, also the unique minimizer of $[P]$.

\section{Numerical characterization of the controls.}

We present next the results from a numerical simulation and the corresponding optimal controls for the resulting problem $[P]$.

We combine a Quasi-Newton (QN) method to compute the optimal solutions of problem $[P]$ with a Finite Difference procedure to approximate the egg, larval and adult density functions of system (2.3). One can prove the convergence of this method in the same way as $\operatorname{in}^{15}$. We consider the analogous discrete adjoint problem, 
that is (using the same notation given in ${ }^{15}$ )

$$
\left\{\begin{aligned}
\lambda_{i_{0}}^{e, n_{0}-1} & =\frac{\Delta t}{\Delta a} \frac{\lambda_{i_{0}+1}^{e, n_{0}} v_{i_{0}}^{e, n_{0}}}{1+\Delta t\left(\beta^{e}+m^{e}\right)_{0}^{n_{0}+1}+\Delta t \alpha_{2} v_{2_{2} i_{0}+1}^{n_{0}+1}}+\lambda_{i_{0}}^{e, n_{0}} \frac{\left(1-\frac{\Delta t}{\Delta a} v_{i_{0}}^{e, n_{0}}\right)}{1+\Delta t\left(\beta^{e}+m^{e}\right)_{i_{0}}^{n_{0}+1}+\Delta t \alpha_{2} v_{2, i_{0}}^{n_{0}+1}} \\
& +\Delta t \frac{\lambda_{1}^{l, n_{0}} \beta_{i_{0}}^{e, n_{0}}}{1+\Delta t\left(\beta^{l}+m^{l}\right)_{1}^{n_{0}+1}}, \\
\lambda_{i_{0}}^{l, n_{0}-1} & =\frac{\Delta t}{\Delta a} \frac{\lambda_{i_{0}+1}^{l, n_{0}} v_{i_{0}}^{l, n_{0}}}{1+\Delta t\left(\beta^{l}+m^{l}\right)_{i_{0}+1}^{n_{0}+1}}+\lambda_{i_{0}}^{l, n_{0}} \frac{\left(1-\frac{\Delta t}{\Delta a} v_{i_{0}}^{l, n_{0}}\right)}{1+\Delta t\left(\beta^{l}+m^{l}\right)_{i_{0}}^{n_{0}+1}}+\Delta t \frac{\lambda_{1}^{f, n_{0}} \beta_{i_{0}}^{l, n_{0}}}{1+\Delta t m_{1}^{f, n_{0}+1}}, \\
\lambda_{i_{0}}^{f, n_{0}-1} & =\frac{\Delta t}{\Delta a} \frac{\lambda_{i_{0}+1}^{f, n_{0}} v_{i_{0}}^{f, n_{0}}}{1+\Delta t m_{i_{0}+1}^{f, n_{0}+1}}+\lambda_{i_{0}}^{f, n_{0}} \frac{\left(1-\frac{\Delta t}{\Delta a} v_{i}^{f, n_{0}}\right)}{1+\Delta t m_{i_{0}}^{f, n} n_{0}+1}+\Delta t \frac{\lambda_{1}^{e, n_{0}}\left(1-\alpha_{1}\left(v_{1}\right)_{i_{0}}^{n_{0}}\right) \beta_{i_{0}}^{f, n_{0}}}{1+\Delta t\left(\beta^{e}+m^{e}\right)_{1}^{n_{0}+1}+\Delta t \alpha_{2}\left(v_{2}\right)_{1}^{n_{0}+1}},
\end{aligned}\right.
$$

for $1 \leq i_{0} \leq N_{a}$ and $1 \leq n_{0} \leq N_{t}$.

The parameters of the model are chosen so that the growth of the population is chronological and constant in time as observed under laboratory conditions ${ }^{16,18}$ (120 eggs per female on average, no mortality and no competition for food) that is

$$
g^{k}(E(t), a) \equiv 1, \quad m^{k}(E(t), a) \equiv 0, \quad \text { for } k=e, l, f,
$$

making the model age-structured; we use truncated normal distributions for the fertility and stage-transition functions given by

$$
\beta^{e}(t, a)=d_{1} e^{-\left(\frac{a-7.5}{0.35}\right)^{2}}=\beta^{l}(t, a), \quad \beta^{f}\left(P^{f}, E(t), a\right)=d_{2} e^{-\left(\frac{a-3.5}{0.35}\right)^{2}},
$$

with $d_{1}=16.12, d_{2}=19.34$, and $L^{e}=L^{f}=L^{l}=10$. In order to be able to present in a single graph the population dynamics of all classes we assume the fertility rate to be one tenth of its actual value, i.e. 12 eggs by female. The only growth variations considered in these examples are inside cohorts as measured and showed in ${ }^{16,18}$.

We computed numerically the optimal control for a population initialized by a cohort of one hundred females with truncated-normal age distribution, that is

$$
u^{f}(0, a)=d_{3} e^{-\left(\frac{a-1}{0.25}\right)^{2}}, \quad d_{3}=225.67 .
$$

The temporal dynamics of these females is represented in Figure 1 by the dotted line, that of their eggs by the solid line, and that of the eggs that become larvae after few days by the dash-dotted line. The total number of larvae during the whole experiment is 1200 individuals.

The financial cost of larval damages per larva per unit of time is assumed to equal one unit, i.e. $\mu=1$, and the the multiplier for the application of ovicide and larvicide is also set equal to unity, i.e. $\eta_{1}=\eta_{2}=1$. For simplicity we take the cost of an ovicide application to be the same as the cost of a mating disruption use, though this is not true in reality. The issue here is not to discuss the actual cost or performance of the pest control methods but rather to show to numerically realize the solution of the optimal control problem $[P]$. 


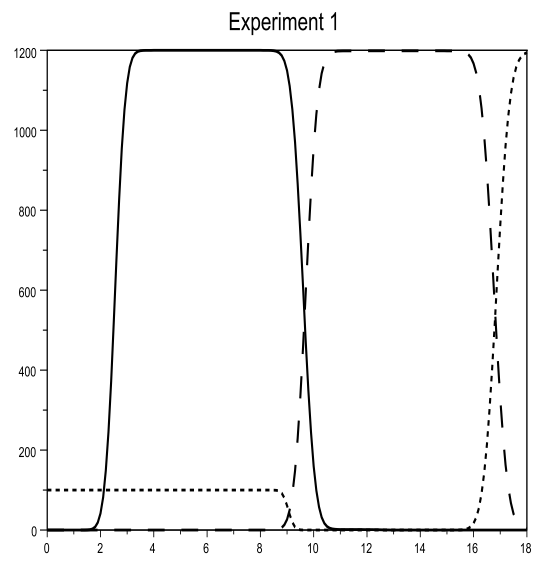

Figure 1. Population dynamics of females (dotted line), eggs (solid line) and larvae (dashed-dotted line) without any control applied. The popluation is initialized with 100 females.

cost or performance of the pest control methods but rather to show to numerically realize the solution of the optimal control problem $[P]$.

We focus on just the first generation of the simulated population dynamics and determine through the optimal control problem $[P]$ presented in Section 2 the best way to use the whole array of chemical products described to minimize the economic damage caused by this insect population. The age- and time-discretization steps are chosen equal to 0.1 and the QN algorithm is initialized with the step-functions represented by the dashed-dotted curves of figure 2 .

The optimal solution computed for problem $[P]$ is given in Figure 2. According to it, the optimal strategy to control a population with dynamics as in Figure 1 is to continuously spray ovicides during the egg laying dynamics (or to combine an egg pesticide application with a use of pheromone dispensers provided that the efficiency of the combination of these chemical products is maximal). In addition to that a larvicide application seems to be required to totally eliminate the insects but, actually, when looking at the integral of this solution $v_{2}$ (cf. Table 1 ) the amount of product is almost null.

Table 1 below shows the contributions to the three components of the cost function for the optimal solution $\left(v_{1}, v_{2}\right)$ of problem $[P]$.

\section{Concluding remarks.}

In this paper we described and analyzed a mathematical model that may realistically guide the "best" choice of control strategies for one of the most ravaging pests 

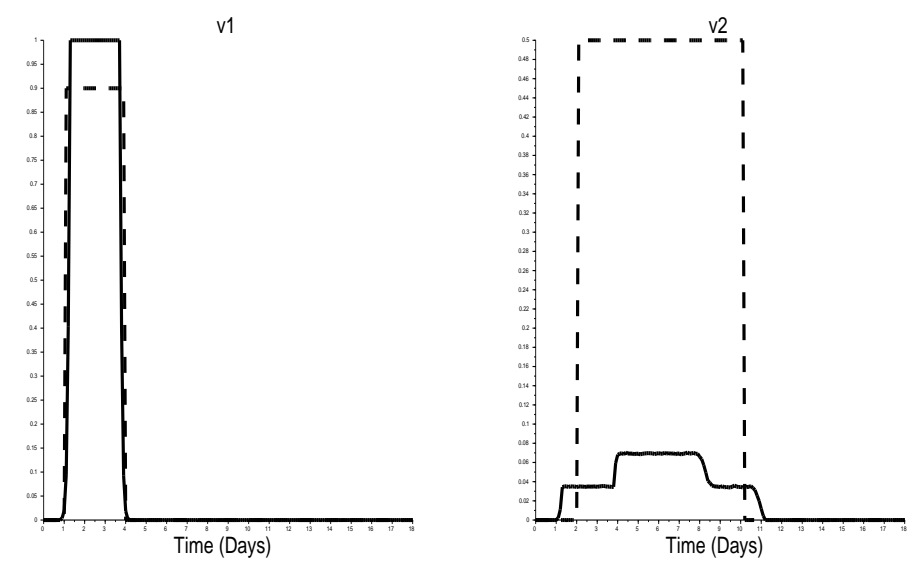

Figure 2. Optimal Control $\left(v_{1}, v_{2}\right.$ in solid curves) for the population of Figure 1, determined from problem $[P]$. The horizontal axis represents the time. The dash-dotted curves are the QN initial functions.

\begin{tabular}{|c|c|c|c|c|}
\hline component & $\mu \int_{0}^{T} P_{\left(v_{1}, v_{2}\right)}^{l}(t) d t$ & $\frac{\eta_{1}}{L^{f}} \int_{0}^{T}\left(v_{1}(t)\right)^{2} d t$ & $\frac{\eta_{2}}{L^{e}} \int_{0}^{T}\left(v_{2}(t)\right)^{2} d t$ & $\mathcal{J}$ \\
\hline value & 0.139 & 0.2534 & 0.00271 & 0.39511 \\
\hline
\end{tabular}

Table 1 . Values of the three integrals that form the cost functional $\mathcal{J}$ at the optimum $\left(v_{1}, v_{2}\right)$ of the control problem $[P]$ presented in Section 2, and the minimum value of the functional $\mathcal{J}$.

that damages the grapes and decreases production in European and American vineyards, specifically that caused by the European grapevine moth (Lobesia botrana).

Our model for control of the pest is based on the Lobesia botrana Model published in ${ }^{2}$. We considered a reduced version of it with modifications to both one of the differential equations and to the boundary condition for the egg density in order to account, respectively, for the application of larval pesticides and that of ovicides and/or pheromones that disrupt and reduce mating and thus reduce egg production too. These three techniques are, in fact, the most widely used in many vineyards in Europe, see ${ }^{8,17,19}$ for example. We constructed a cost function that is amenable to matching with real-life financial costs by allowing linear dependence on the density of the pest and nonlinear dependence on the control. This is in sharp contrast with the majority of models for optimal control where the dependence of the cost on the relevant density and on the control is quadratic so that classical techniques based on a Lagrangian and a dual problem can be applied to show existence of an optimal control. This is one of the novel features in our results, and one that is very desirable when trying to apply a mathematical model to a real-life situation. 
tration of the optimal control pair that consists of continous functions for a given population dynamics.

These results provide a solid ground on which vineyard managers can base a treatment protocol for this pest that will minimize financial losses. Even though our model includes the possible use of mating disruption as a control measure, difficulties arise when considering its efficiency. On the other hand, while the use of pesticides has a clear direct financial cost given by the purchase and application costs for the chemical, it also has another cost, more subtle and harder to model and evaluate, that we could refer to as "environmental cost" due to the poisoning of the soil. We shall address these and other issues related to the modeling of the coefficients in the control terms and in the cost function in a forthcoming paper.

\section{Bibliography}

1. B. Ainseba, S. Anita, M. Langlais, Optimal control for a nonlinear age-structured population dynamics model, Electronic Journal of Differential Equations, 28: 1-9 (2002).

2. B. Ainseba, D. Picart, An innovative multistage, physiologically structured, population model to understand the European grapevine moth dynamics. Journal of Mathematical analysis and Applications, 382: 34-46(2011).

3. V. Barbu and M. Iannelli, Optimal Control of Population Dynamics. Journal of optimization theory and applications 102: 1-14 (1999)

4. G. Busoni, S. Matuccia, Problem of Optimal Harvesting Policy in Two-Stage AgeDependent Populations. Mathematical Biosciences, 143: 1-33 (1997).

5. European Grapevine Moth (EGVM), California Department of Food and Drugs, http://www.cdfa.ca.gov/plant/egvm/index.html

6. J.V. Cross, D.R. Hall, Exploitation of the sex pheromone of apple leaf midge Dasineura mali Kieffer (Diptera: Cecidomyiidae) for pest monitoring: Part1. development of lure and trap. Crop Protection, 28: 139-144 (2009).

7. I. Ekeland, On the variational principle, Journal Math. Appl. 47: 324-353 (1974).

8. D. Esmenjaud, S. Kreiter, M. Martinez, R. Sforza, D. Thiéry, M. Van Helden, M. Yvon, Ravageurs de la vigne, Éditions Féret, Bordeaux (2008).

9. K. R. Fister and S. Lenhart, Optimal Harvesting in an Age-Structured Predator-Prey Model. Applied Mathematics and Optimization, 54:1-15 (2006).

10. K. R. Fister, S. Lenhart, Optimal control of a competitive system with age-structure. Journal of Mathematical Analysis and Applications, 291: 526-537 (2004).

11. H. R. Joshi, S. Lenhart, H. Lou, H. G., Harvesting control in an integrodifference population model with concave growth term. Nonlinear Analysis: Hybrid Systems, 1: 417-429 (2007).

12. S. Lenhart, M. Liang and V. Protopopescu, Optimal Control of Boundary Habitat Hostility for Interacting Species. Mathematical Methods in the Applied Sciences, 22: 1061-1077 (1999).

13. E.S. Martins, L.B. Praça, V.F. Dumas, J.O. Silva-Werneck, E.H. Sone, I.C. Waga, C. berry, R.G. Monnerat, Characterization of Bacillus thuringiensis isolates toxic to cotton boll weevil (Anthonomus grandis). Biological Control, 40: 65-68 (2005).

14. D. Picart, B. Ainseba, F. Milner, Optimal control problem on insect pest populations. Applied Mathematics Letters, 24: 1160-1164 (2011).

15. D. Picart, B. Ainseba, Parameter identification in multistage population dynamics model. Nonlinear Analysis: Real World Applications, 12: 3315-3328 (2011).

16. D. Picart, Modélisation et estimation des paramètres liés au succès reproducteur dun 
ravageur de la vigne (Lobesia botrana DEN. \& SCHIFF), PhD Thesis, No. 3772 University of Bordeaux, 2009.

17. R. Roehrich, J.P. Carles, C. Tresor, M.A. de Vathaire, Essais de confusion sexuelle contre les tordeuses de la grappe, l'eudemis Lobesia botrana Den. et Schiff. et la cochylis Eupoecilia ambiguella Hb. Annales de Zoologie et d'Ecololgie Animales, 11: 659-675 (1979).

18. D. Thiéry, J. Moreau, Relative performance of European grapevine moth (Lobesia botrana) on grapes and other hosts. Oecologia 143: 548-557 (2005).

19. V.A. Vassiliou, Control of Lobesia botrana (Lepidoptera: Tortricidae) in vineyards in Cyprus using the Mating Disruption Technique, Crop Protection 28: 145-150 (2009).

20. C.R. Weeden, A. M. Shelton, M. P. Hoffman, Biological Control: A Guide to Natural Enemies in North America, http://www.nysaes.cornell.edu/ent/biocontrol/

21. C. Zhao, M. Wang, P. Zhao, Optimal Control of Harvesting for Age-Dependent Predator-Prey System. Mathematical and Computer Modelling, 42: 573-584 (2005).

\section{Appendix.}

For the proofs presented in the following we make some additional hypotheses. The growth functions $g^{k}$, for $k=e, l, f$, are non-negative and bounded away from zero at the initial age 0 ,

$$
0<\underline{g}^{k} \leq g^{k}(E(t), 0) \leq \bar{g}^{k}, \quad k=e, l, f
$$

The birth function $\beta^{f}\left(P^{f}, t, a\right)$ is differentiable in the first variable $P^{f}$ and its derivative is Lipschitz continuous with constant $\beta_{K}$ such that

$$
\begin{array}{r}
\left|\beta^{f}\left(P_{v}^{f}, E(t), a\right)-\beta^{f}\left(P_{\tilde{v}}^{f}, E(t), a\right)\right|+\mid \partial_{x} \beta^{f}\left(P_{v}^{f}, E(t), a\right)- \\
-\partial_{x} \beta^{f}\left(P_{\tilde{v}}^{f}, E(t), a\right) \mid \\
\leq \beta_{K}\left\|u_{v}^{f}-u_{\tilde{v}}^{f}\right\|_{L^{1}},
\end{array}
$$

where $P_{v}^{f}(t)=\int_{0}^{L^{f}} u_{v}^{f}(t, a) d a$ and $u_{v}^{f}$ satisfies (2.3) with the control $v$.

The larval mortality function $m^{l}\left(P^{l}, E(t), a\right)$ is differentiable in the first variable $P^{l}$ and its derivative is Lipschitz continuous with constant $m_{K}$ such that

$$
\begin{array}{r}
\left|m^{l}\left(P_{v}^{l}, E(t), a\right)-m^{l}\left(P_{\tilde{v}}^{l}, E(t), a\right)\right|+\left|\partial_{x} m^{l}\left(P_{v}^{l}, E(t), a\right)-\partial_{x} m^{l}\left(P_{\tilde{v}}^{l}, E(t), a\right)\right| \\
\leq m_{K}\left\|u_{v}^{l}-u_{\tilde{v}}^{l}\right\|_{L^{1}},
\end{array}
$$

where $P_{v}^{l}(t)=\int_{0}^{L^{l}} u_{v}^{l}(t, a) d a$ and $u_{v}^{l}$ satisfies (2.3) with the control $v$.

\subsection{Continuity of the state variables with respect to the controls.}

Proof of theorem 1: Let us introduce the following change of variables,

$$
\hat{u}_{v}^{k}(t, a)=e^{-\lambda t} u_{v}^{k}(t, a), \quad(t, a) \in \Omega^{k}, \quad k=e, l, f,
$$


where $u_{v}^{k}$ satisfies (2.3) and the new variables $\hat{u}_{v}^{k}$ satisfy the following system,

$$
\left\{\begin{array}{c}
\begin{array}{rl}
\frac{\partial}{\partial t} \hat{u}_{v}^{e}(t, a)+\frac{\partial}{\partial a}\left[g^{e}(E(t), a) \hat{u}_{v}^{e}(t, a)\right]+\lambda \hat{u}_{v}^{e}(t, a) & +\left(\beta^{e}(E(t), a)+m^{e}(E(t), a)\right) \hat{u}_{v}^{e}(t, a) \\
& +\alpha_{2} v_{2}(t, a) \hat{u}_{v}^{e}(t, a)=0 \\
\frac{\partial}{\partial t} \hat{u}_{v}^{l}(t, a)+\frac{\partial}{\partial a}\left[g^{l}(E(t), a) \hat{u}_{v}^{l}(t, a)\right]+\lambda \hat{u}_{v}^{l}(t, a)+\beta^{l}(E(t), a) \hat{u}_{v}^{l}(t, a) & +m^{l}\left(P^{l}, E(t), a\right) \hat{u}_{v}^{l}(t, a)=0 \\
\frac{\partial}{\partial t} \hat{u}_{v}^{f}(t, a)+\frac{\partial}{\partial a}\left[g^{f} \hat{u}_{v}^{f}(t, a)\right]+\lambda \hat{u}_{v}^{f}(t, a)+m^{f}(E(t), a) \hat{u}_{v}^{f}(t, a)=0 \\
g^{e}(E(t), 0) \hat{u}_{v}^{e}(t, 0)=\int_{0}^{L^{f}}\left(1-\alpha_{1} v_{1}(t, a)\right) \beta^{f}\left(P^{f}, E(t), a\right) \hat{u}_{v}^{f}(t, a) d a \\
g^{l}(E(t), 0) \hat{u}_{v}^{l}(t, 0)=\int_{0}^{L^{e}} \beta^{e}(E(t), a) \hat{u}_{v}^{e}(t, a) d a
\end{array} \\
g^{f}(E(t), 0) \hat{u}_{v}^{f}(t, 0)=\int_{0}^{L^{l}} \beta^{l}(E(t), a) \hat{u}_{v}^{l}(t, a) d a \\
\hat{u}_{v}^{k}(0, a)=e^{-\lambda t} u_{0}^{k}(a), \quad k=e, l, f .
\end{array}\right.
$$

Now, let $v$ and $\tilde{v}$ be two controls and let $\hat{u}_{v}^{k}$ and $\hat{u}_{\tilde{v}}^{k}$, respectively, be the corresponding solutions of (7.1), for $k=e, l, f$. The corresponding differences between these solutions, denoted $\hat{u}^{k}$, satisfy the system (7.2) below,

$$
\left\{\begin{array}{c}
\begin{array}{rl}
\frac{\partial}{\partial t} \hat{u}^{e}(t, a)+\frac{\partial}{\partial a}\left[g^{e}(E(t), a) \hat{u}^{e}(t, a)\right] & +\lambda \hat{u}^{e}(t, a)+\left(\beta^{e}(E(t), a)+m^{e}(E(t), a)\right) \hat{u}^{e} \\
& +\alpha_{2} \tilde{v}_{2}(t, a) \hat{u}^{e}+\alpha_{2}\left(v_{2}(t, a)-\tilde{v}_{2}(t, a)\right) \hat{u}_{v}^{e}=0, \\
\frac{\partial}{\partial t} \hat{u}^{l}(t, a)+\frac{\partial}{\partial a}\left[g^{l}(E(t), a) \hat{u}^{l}(t, a)\right] & +\lambda \hat{u}^{l}(t, a)+\left(\beta^{l}(E(t), a)+m_{v}^{l}\right) \hat{u}^{l}(t, a) \\
& +\left(m_{v}^{l}-m_{\tilde{v}}^{l}\right) u_{\tilde{v}}^{l}=0,
\end{array} \\
\begin{array}{rl}
\frac{\partial}{\partial t} \hat{u}^{f}(t, a)+\frac{\partial}{\partial a}\left[g^{f}(E(t), a) \hat{u}^{f}(t, a)\right]+\lambda \hat{u}^{f}(t, a)+m^{f}(E(t), a) \hat{u}^{f}(t, a)=0, \\
g^{e}(E(t), 0) \hat{u}^{e}(t, 0)=\int_{0}^{L^{f}}\left(1-\alpha_{1} \tilde{v}_{1}(t, a)\right) \beta_{v}^{f} \hat{u}^{f}(t, a) d a
\end{array} \\
\quad-\int_{0}^{L^{f}} \alpha_{1}\left(v_{1}-\tilde{v}_{1}\right)(t, a) \beta_{v}^{f} \hat{u}_{v}^{f}(t, a) d a+\int_{0}^{L^{f}}\left(1-\alpha_{1} \tilde{v}_{1}(t, a)\right)\left(\beta_{v}^{f}-\beta_{\tilde{v}}^{f}\right) \hat{u}_{\tilde{v}}^{f}(t, a) d a, \\
\left.g^{l}(E(t), 0) \hat{u}^{l}\right)(t, 0)=\int_{0}^{L^{e}} \beta^{e}(E(t), a) \hat{u}^{e}(t, a) d a, \\
g^{f}(E(t), 0) \hat{u}^{f}(t, 0)=\int_{0}^{L^{l}} \beta^{l}(E(t), a) \hat{u}^{l}(t, a) d a, \\
\hat{u}^{k}(0, a)=0, \quad k=e, l, f
\end{array}\right.
$$

where $m_{v}^{l}=m^{l}\left(P_{v}^{l}, E(t), a\right)$ and $\beta_{v}^{f}=\beta^{f}\left(P_{v}^{f}, E(t), a\right)$. We multiply the first, second and third equations of (7.2), respectively, by $\hat{u}^{e}, \hat{u}^{l}$ and $\hat{u}^{f}$, then we integrate them on the corresponding domain $\Omega^{k}$ to get the following system,

$$
\left\{\begin{array}{c}
\lambda \int_{\Omega^{e}}\left(\hat{u}^{e}\right)^{2}(t, a) d a d t-\frac{1}{2} \int_{0}^{T} g^{e}(E(t), 0)\left(\hat{u}^{e}(t, 0)\right)^{2} d t \\
\quad \leq \int_{\Omega^{e}}\left[\tilde{v}_{2}(t, a)-v_{2}(t, a)\right] \hat{u}_{v}^{e}(t, a) \hat{u}^{e}(t, a) d a d t \\
\lambda \int_{\Omega^{l}}\left(\hat{u}^{l}\right)^{2}(t, a) d a d t-\frac{1}{2} \int_{0}^{T} g^{l}(E(t), 0)\left(\hat{u}^{l}(t, 0)\right)^{2} d t \\
\quad \leq \int_{\Omega^{l}}\left(m_{v}^{l}-m_{\tilde{v}}^{l}\right) \hat{u}_{\tilde{v}}^{l}(t, a) \hat{u}^{l}(t, a) d a d t \\
\lambda \int_{\Omega^{f}}\left(\hat{u}^{f}\right)^{2}(t, a) d a d t-\frac{1}{2} \int_{0}^{T} g^{f}(E(t), 0)\left(\hat{u}^{f}(t, 0)\right)^{2} d t \leq 0 .
\end{array}\right.
$$


These can be modified by applying Young's inequality to the integrals of the first and second equations on the right side to obtain system (7.3).

$$
\left\{\begin{array}{c}
\begin{array}{r}
\lambda \int_{\Omega^{e}}\left(\hat{u}^{e}\right)^{2}(t, a) d a d t \leq \frac{1}{2} \int_{0}^{T} g^{e}(E(t), 0)\left(\hat{u}^{e}(t, 0)\right)^{2} d t+\frac{\epsilon_{1}\left\|\hat{u}_{v}^{e}\right\|_{L^{2}}^{2}}{2} \int_{\Omega^{e}}\left(\hat{u}^{e}\right)^{2}(t, a) d a d t \\
\quad+\frac{1}{2 \epsilon_{1}} \int_{\Omega^{e}}\left|\tilde{v}_{2}(t, a)-v_{2}(t, a)\right|^{2} d a d t
\end{array} \\
\lambda \int_{\Omega^{l}}\left(\hat{u}^{l}\right)^{2}(t, a) d a d t \leq \frac{1}{2} \int_{0}^{T} g^{l}(E(t), 0)\left(\hat{u}^{l}(t, 0)\right)^{2} d t+m_{K} L^{l}\left\|\hat{u}_{v}^{l}\right\|_{L^{2}} \int_{\Omega^{l}}\left(\hat{u}^{l}\right)^{2}(t, a) d a d t \\
\lambda \int_{\Omega^{f}}\left(\hat{u}^{f}\right)^{2}(t, a) d a d t \leq \frac{1}{2} \int_{0}^{T} g^{f}(E(t), 0)\left(\hat{u}^{f}(t, 0)\right)^{2} d t .
\end{array}\right.
$$

Next we use the boundary conditions given in (7.2) for the densities $\hat{u}^{k}(t, 0)(k=$ $e, l, f)$ to evaluate the first integrals on the right hand sides of the three inequalities above to obtain

$$
\left\{\begin{array}{l}
\frac{1}{2} \int_{0}^{T} g^{e}(E(t), 0)\left(\hat{u}^{e}(t, 0)\right)^{2} d t=\frac{1}{2} \int_{0}^{T} \frac{1}{g^{e}(E(t), 0)}\left[\int_{0}^{L^{f}}\left(1-\alpha_{1} \tilde{v}_{1}(t, a)\right) \beta_{v}^{f} \hat{u}^{f}(t, a) d a\right. \\
\left.\quad+\alpha_{1} \int_{0}^{L}\left(\tilde{v}_{1}-v_{1}\right)(t, a) \beta_{v}^{f} \hat{u}_{v}^{f}(t, a) d a+\int_{0}^{L^{f}}\left(1-\alpha_{1} \tilde{v}_{1}(t, a)\right)\left(\beta_{v}^{f}-\beta_{\tilde{v}}^{f}\right) \hat{u}_{\tilde{v}}^{f}(t, a) d a\right]^{2} d t, \\
\frac{1}{2} \int_{0}^{T} g^{l}(E(t), 0)\left(\hat{u}^{l}(t, 0)\right)^{2} d t=\frac{1}{2} \int_{0}^{T} \frac{1}{g^{l}(t, 0)}\left[\int_{0}^{L^{e}} \beta^{e}(t, a) \hat{u}^{e}(t, a) d a\right]^{2} d t, \\
\frac{1}{2} \int_{0}^{T} g^{f}(E(t), 0)\left(\hat{u}^{f}(t, 0)\right)^{2} d t=\frac{1}{2} \int_{0}^{T} \frac{1}{g^{f}(t, 0)}\left[\int_{0}^{L^{l}} \beta^{l}(t, a) \hat{u}^{l}(t, a) d a\right]^{2} d t .
\end{array}\right.
$$

Developing the squares in the above expressions, and applying Young's and CauchySwartz's inequalities, we see that

$$
\left\{\begin{array}{l}
\frac{1}{2} \int_{0}^{T} g^{e}(E(t), 0)\left(\hat{u}^{e}(t, 0)\right)^{2} d t \leq \frac{1}{2 \underline{g}^{e}} \int_{0}^{T}\left[\left\|\beta^{f}\right\|_{L^{2}}^{2}+2 \bar{\beta}^{f} \beta_{K} L^{f}\left\|\hat{u}_{\tilde{v}}^{f}(t)\right\|_{L^{1}}\right. \\
\left.\quad+\left(\beta_{K}\right)^{2} L^{f}\left\|\hat{u}_{\tilde{v}}^{f}(t)\right\|_{L^{1}}^{2}+\epsilon_{2}\left\|\beta^{f}\right\|_{L^{2}}^{2}+\epsilon_{3} L^{f} \beta_{K}\left\|\hat{u}_{\tilde{v}}^{f}(t)\right\|_{L^{1}}\right] \int_{0}^{L^{f}}\left(\hat{u}^{f}\right)^{2}(t, a) d a d t \\
\quad+\frac{\left(\bar{\beta}^{f}\right)^{2}}{2 \underline{g}^{e}}\left\|\hat{u}_{v}^{f}(t)\right\|_{L^{2}}^{2}\left[\frac{1}{\epsilon_{2}}+\frac{\beta_{K}}{\epsilon_{3}}+1\right] \int_{\Omega^{f}}\left[\tilde{v}_{1}(t, a)-v_{1}(t, a)\right]^{2} d a d t \\
\frac{1}{2} \int_{0}^{T} g^{l}(E(t), 0)\left(\hat{u}^{l}(t, 0)\right)^{2} d t \leq \frac{\left\|\beta^{e}\right\|_{L^{2}}^{2}}{2 \underline{g}^{l}} \int_{\Omega^{e}}\left(\hat{u}^{e}\right)^{2}(t, a) d a d t \\
\frac{1}{2} \int_{0}^{T} g^{f}(E(t), 0)\left(\hat{u}^{f}(t, 0)\right)^{2} d t \leq \frac{\left\|\beta^{l}\right\|_{L^{2}}^{2}}{2 \underline{g}^{f}} \int_{\Omega^{l}}\left(\hat{u}^{l}\right)^{2}(t, a) d a d t .
\end{array}\right.
$$


We now substitute the estimates (7.4) into (7.3) to obtain the following inequalities:

$$
\left\{\begin{array}{l}
\left(\lambda-\frac{\epsilon_{1}\left\|\hat{u}_{v}^{e}\right\|_{L^{2}}^{2}}{2}\right) \int_{\Omega^{e}}\left(\hat{u}^{e}\right)^{2}(t, a) d a d t \leq \frac{A}{2 \underline{g}^{e}} \int_{\Omega^{f}}\left(\hat{u}^{f}\right)^{2}(t, a) d a d t \\
\quad+\frac{B}{2 \underline{g}^{e}} \int_{\Omega^{f}}\left|\tilde{v}_{1}(t, a)-v_{1}(t, a)\right|^{2} d a d t+\frac{1}{2 \epsilon_{1}} \int_{\Omega^{f}}\left|\tilde{v}_{2}(t, a)-v_{2}(t, a)\right|^{2} d a d t \\
\left(\lambda-m_{K} L^{l}\left\|\hat{u}_{v}^{l}\right\|_{L^{2}}\right) \int_{\Omega^{l}}\left(\hat{u}^{l}\right)^{2}(t, a) d a d t \leq \frac{\left\|\beta^{e}\right\|_{L^{2}}^{2}}{2 \underline{g}^{l}} \int_{\Omega^{e}}\left(\hat{u}^{e}\right)^{2}(t, a) d a d t \\
\lambda \int_{\Omega^{f}}\left(\hat{u}^{f}\right)^{2}(t, a) d a d t \leq \frac{\left\|\beta^{l}\right\|_{L^{2}}^{2}}{2 \underline{g}^{f}} \int_{\Omega^{l}}\left(\hat{u}^{l}\right)^{2}(t, a) d a d t,
\end{array}\right.
$$

where

$$
\begin{array}{r}
A=\left[\left\|\beta^{f}\right\|_{L^{2}}^{2}+2 \bar{\beta}^{f} \beta_{K} L^{f}\left\|\hat{u}_{\tilde{v}}^{f}(t)\right\|_{L^{1}}+\left(\beta_{K}\right)^{2} L^{f}\left\|\hat{u}_{\tilde{v}}^{f}(t)\right\|_{L^{1}}^{2}+\epsilon_{2}\left\|\beta^{f}\right\|_{L^{2}}^{2}\right. \\
\left.+\epsilon_{3} L^{f} \beta_{K}\left\|\hat{u}_{\tilde{v}}^{f}(t)\right\|_{L^{1}}\right]>0,
\end{array}
$$

and,

$$
B=\left(\bar{\beta}^{f}\right)^{2}\left\|\hat{u}_{v}^{f}(t)\right\|_{L^{2}}^{2}\left[\frac{1}{\epsilon_{2}}+\frac{\beta_{K}}{\epsilon_{3}}+1\right]>0
$$

If we now set

$$
\epsilon_{1}=\frac{2(\lambda-1)}{\left\|\hat{u}_{v}^{e}\right\|_{L^{2}}^{2}}, \quad \epsilon_{2}=1, \quad \epsilon_{3}=1
$$

and assume $\lambda>m_{K}^{l} L^{f}\left\|\hat{u}_{v}^{l}\right\|_{L^{2}}$ and it also satisfies the condition

$$
\lambda\left(\lambda-m_{K} L^{l}\left\|\hat{u}_{v}^{l}\right\|_{L^{2}}\right)>\frac{A\left\|\beta^{l}\right\|_{L^{2}}^{2}\left\|\beta^{e}\right\|_{L^{2}}^{2}}{8 \underline{g}^{e} \underline{g}^{f} \underline{g}^{l}}>0,
$$

then, from (7.5) we derive the following inequality:

$$
\left\{\begin{array}{l}
\int_{\Omega^{e}}\left(\hat{u}^{e}\right)^{2}(t, a) d a d t \leq \\
\quad \frac{C^{-1} B}{2 \underline{g}^{e}} \int_{\Omega^{f}}\left|\tilde{v}_{1}(t, a)-v_{1}(t, a)\right|^{2} d a d t+\frac{C^{-1}}{2 \epsilon_{1}} \int_{\Omega^{f}}\left|\tilde{v}_{2}(t, a)-v_{2}(t, a)\right|^{2} d a d t,
\end{array}\right.
$$

where

$$
C=\left(1-\frac{A}{2 \lambda \underline{g}^{e}} \frac{\left\|\beta^{l}\right\|_{L^{2}}^{2}}{2 \underline{g}^{f}} \frac{\left\|\beta^{e}\right\|_{L^{2}}^{2}}{2 \underline{g}^{l}} \frac{1}{\left(\lambda-m_{K} L^{l}\left\|\hat{u}_{v}^{l}\right\|_{L^{2}}\right)}\right),
$$

and it then follows that the $L^{2}$-norm of the density $\hat{u}^{e}$ can be bounded as follows:

$$
\left\|\hat{u}^{e}\right\|_{L^{2}\left(\Omega^{e}\right)} \leq C^{-1 / 2}\left[\left(\frac{B}{2 \underline{g}^{e}}\right)^{2}+\left(\frac{\left\|\hat{u}_{v}^{e}\right\|_{L^{2}}^{2}}{4(\lambda-1)}\right)^{2}\right]^{1 / 2}\|\tilde{v}-v\|_{L^{\infty}} .
$$


Finally, we conclude the proof of theorem 1 for $k=e$ using the constants

$$
\begin{aligned}
& D_{1}^{e}=L T\left(\frac{B}{2 C \underline{g}^{e}}\right)^{1 / 2} \\
& D_{2}^{e}=\frac{L T\left\|\hat{u}_{v}^{e}\right\|_{L^{2}}}{2}\left[\frac{1}{C(\lambda-1)}\right]^{1 / 2}
\end{aligned}
$$

where the constants $B$ and $C$ are defined, respectively, in (7.6) and (7.8), and $\lambda$ satisfies the condition (7.7).

Similarly, in order to derive the two other inequalities of theorem 1 (for $k=e, l$ ) we use the inequalities (7.5) with the above definition of $\epsilon_{i}, i=1,2,3$, to finally conclude that the corresponding constants $D^{l}$ and $D^{f}$ are given by

$$
\begin{aligned}
& D_{1}^{l}=\frac{L T\left\|\beta^{e}\right\|_{L^{2}}}{2}\left[\left(\frac{B}{\underline{g}^{e} \underline{g}^{l}}\right)\left(\frac{C^{-1}}{\lambda-m_{K} L^{l}\left\|\hat{u}_{v}^{l}\right\|_{L^{2}}}\right)\right]^{1 / 2}, \\
& D_{2}^{l}=\frac{L T\left\|\beta^{e}\right\|_{L^{2}}\left\|\hat{u}_{v}^{e}\right\|_{L^{2}}}{2}\left[\left(\frac{1}{\underline{g}^{l}(\lambda-1)}\right)\left(\frac{C^{-1}}{\lambda-m_{K} L^{l}\left\|\hat{u}_{v}^{l}\right\|_{L^{2}}}\right)\right]^{1 / 2}, \\
& D_{1}^{f}=\frac{L T\left\|\beta^{e}\right\|_{L^{2}}\left\|\beta^{l}\right\|_{L^{2}}}{2}\left[\left(\frac{B}{2 \underline{g}^{e} \underline{g}^{l} \underline{g}^{f}}\right)\left(\frac{(\lambda C)^{-1}}{\lambda-m_{K} L^{l}\left\|\hat{u}_{v}^{l}\right\|_{L^{2}}}\right)\right]^{1 / 2}, \\
& D_{2}^{f}=\frac{L T\left\|\beta^{e}\right\|_{L^{2}}\left\|\beta^{l}\right\|_{L^{2}}\left\|\hat{u}_{v}^{e}\right\|_{L^{2}}}{2}\left[\left(\frac{1}{g^{l} \underline{g}^{f}(\lambda-1)}\right)\left(\frac{(\lambda C)^{-1}}{\lambda-m_{K} L^{l}\left\|\hat{u}_{v}^{l}\right\|_{L^{2}}}\right)\right]^{1 / 2},
\end{aligned}
$$

and $\lambda$ satisfies the condition (7.7).

\subsection{Continuity of the dual variables with respect to the controls.}

Before giving the proof of theorem 3 we shall estimate the $L^{2}$-norm of the dual variable $p^{e}$.

Let us introduce the following change of variables,

$$
\hat{p}_{v}^{k}(t, a)=e^{\lambda t} p_{v}^{k}(t, a) \quad k=e, l, f
$$

where $p_{v}^{k}$ satisfies (4.1) with the control $v=\left(v_{1}, v_{2}\right)$, and $\hat{p}_{v}^{k}$ satisfies system (7.9) 
below.

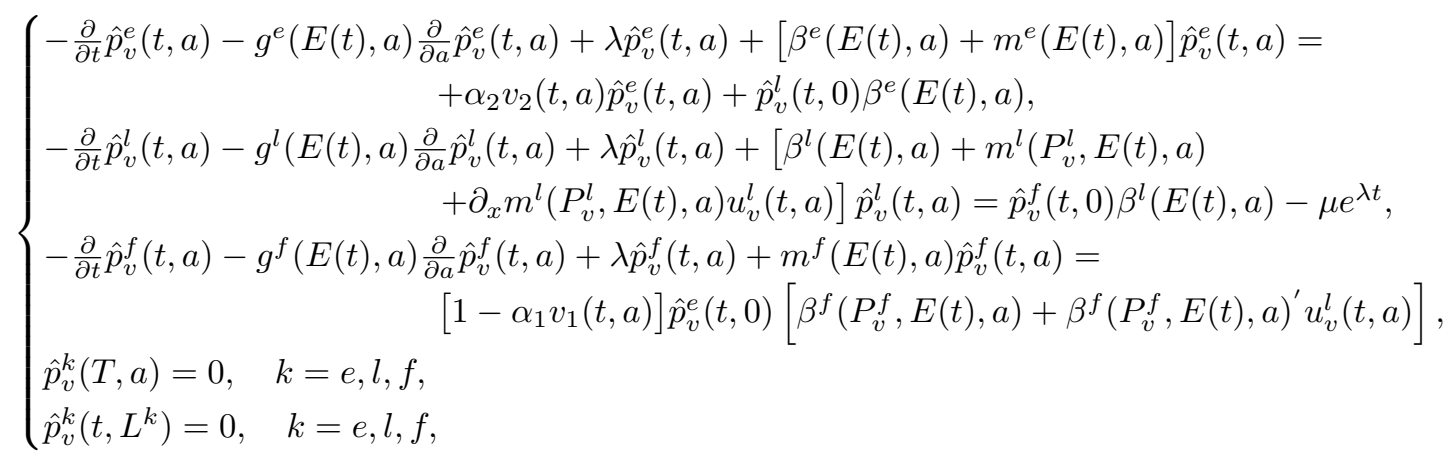

where $P_{v}^{k}(t)=\int_{0}^{L^{k}} u_{v}^{k}(t, a) d a, k=l, f$, and $u_{v}^{k}$ is given by (2.3). We next multiply the first three equations of (7.9), respectively by $\hat{p}_{v}^{e}, \hat{p}_{v}^{l}$ and $\hat{p}_{v}^{f}$, and then we integrate the resulting equations on the corresponding sets $\Omega^{k}(k=e, l, f)$ to see that

$$
\left\{\begin{array}{r}
\frac{1}{2} \int_{0}^{T}\left(\hat{p}_{v}^{e}\right)^{2}(t, 0) d t+\lambda \int_{\Omega^{e}}\left(\hat{p}_{v}^{e}\right)^{2}(t, a) d a d t \leq \int_{\Omega^{e}} \hat{p}_{v}^{l}(t, 0) \beta^{e}(E(t), a) \hat{p}_{v}^{e}(t, a) d a d t \\
\frac{1}{2} \int_{0}^{T}\left(\hat{p}_{v}^{l}\right)^{2}(t, 0) d t+\lambda \int_{\Omega^{l}}\left(\hat{p}_{v}^{l}\right)^{2}(t, a) d a d t \leq \int_{\Omega^{l}} \hat{p}_{v}^{f}(t, 0) \beta^{l}(E(t), a) \hat{p}_{v}^{l}(t, a) d a d t \\
\begin{array}{r}
\frac{1}{2} \int_{0}^{T}\left(\hat{p}_{v}^{f}\right)^{2}(t, 0) d t+\lambda \int_{\Omega^{f}}\left(\hat{p}_{v}^{f}\right)^{2}(t, a) d a d t \leq \int_{\Omega^{f}} \hat{p}_{v}^{e}(t, 0)\left[\beta^{f}\left(P^{f}, E(t), a\right)\right. \\
\left.+\beta^{f}\left(P^{f}, E(t), a\right)^{\prime} u^{l}(t, a)\right] \hat{p}_{v}^{f}(t, a) d a d t .
\end{array}
\end{array}\right.
$$

We apply now Young's and Cauchy Schwartz's inequalities to the right-hand sides of the above inequalities to obtain the bounds

$$
\left\{\begin{aligned}
& \int_{\Omega^{e}} \hat{p}_{v}^{l}(t, 0) \beta^{e}(E(t), a) \hat{p}_{v}^{e}(t, a) d a d t \leq \frac{\epsilon_{1}\left\|\beta^{e}\right\|_{L^{2}}^{2}}{2} \int_{\Omega^{e}}\left(\hat{p}_{v}^{e}\right)^{2}(t, a) d a d t \\
& \int_{\Omega^{l}} \hat{p}_{v}^{f}(t, 0) \beta^{l}(E(t), a) \hat{p}_{v}^{l}(t, a) d a d t \leq \frac{1}{2 \epsilon_{1}} \int_{0}^{T^{2}}\left(\hat{p}_{v}^{l}\right)^{2}(t, 0) d t \\
& 2 \int_{L^{2}}^{2}\left(\hat{p}_{v}^{l}\right)^{2}(t, a) d a d t \\
&+\frac{1}{2 \epsilon_{3}} \int_{0}^{T}\left(\hat{p}_{v}^{f}\right)^{2}(t, 0) d t, \\
& \int_{\Omega^{f}} \hat{p}_{v}^{e}(t, 0)\left[\beta^{f}\left(P^{f}, E(t), a\right)+\beta^{f}\left(P^{f}, E(t), a\right)^{\prime} u_{v}^{l}(t, a)\right] \hat{p}_{v}^{f}(t, a) d a d t \\
& \leq\left(\frac{\epsilon_{5}\left\|\beta^{f}\right\|_{L^{2}}^{2}}{2}+\frac{\epsilon_{6} \beta_{P}^{f}\left\|u_{v}^{l}(t)\right\|_{L^{2}}^{2}}{2}\right) \int_{\Omega^{f}}\left(\hat{p}_{v}^{f}\right)^{2}(t, a) d a d t \\
&+\left(\frac{1}{2 \epsilon_{5}}+\frac{\beta_{P}^{f}}{2 \epsilon_{6}}\right) \int_{0}^{T}\left(\hat{p}_{v}^{e}\right)^{2}(t, 0) d t .
\end{aligned}\right.
$$


Next, by setting

$$
\epsilon_{1}=\frac{\lambda-1}{\left\|\beta^{e}\right\|_{L^{2}}^{2}}, \quad \epsilon_{3}=\frac{2 \lambda}{\left\|\beta^{l}\right\|_{L^{2}}^{2}}, \quad \epsilon_{5}=\frac{2(\lambda-1)}{\left\|\beta^{f}\right\|_{L^{2}}^{2}}, \quad \epsilon_{6}=\frac{2}{\left\|u_{v}^{l}(t)\right\|_{L^{2}}^{2}},
$$

system (7.10) becomes

$$
\left\{\begin{array}{l}
\frac{1}{2} \int_{0}^{T}\left(\hat{p}_{v}^{e}\right)^{2}(t, 0) d t+\int_{\Omega^{e}}\left(\hat{p}_{v}^{e}\right)^{2}(t, a) d a d t \leq \frac{1}{2 \epsilon_{1}} \int_{0}^{T}\left(\hat{p}_{v}^{l}\right)^{2}(t, 0) d t \\
\frac{1}{2} \int_{0}^{T}\left(\hat{p}_{v}^{l}\right)^{2}(t, 0) d t \leq \frac{1}{2 \epsilon_{3}} \int_{0}^{T}\left(\hat{p}_{v}^{f}\right)^{2}(t, 0) d t \\
\frac{1}{2} \int_{0}^{T}\left(\hat{p}_{v}^{f}\right)^{2}(t, 0) d t \leq\left(\frac{1}{2 \epsilon_{5}}+\frac{\beta_{P}^{f}}{2 \epsilon_{6}}\right) \int_{0}^{T}\left(\hat{p}_{v}^{e}\right)^{2}(t, 0) d t
\end{array}\right.
$$

that leads immediately to the single inequality

$$
\int_{\Omega^{e}}\left(\hat{p}_{v}^{e}\right)^{2}(t, a) d a d t \leq \frac{1}{2 \epsilon_{1} \epsilon_{3}}\left(\frac{1}{\epsilon_{5}}+\frac{\beta_{P}^{f}}{\epsilon_{6}}\right) \int_{0}^{T}\left(\hat{p}_{v}^{e}\right)^{2}(t, 0) d t .
$$

If $\lambda>1$ and $\lambda$ satisfies the condition

$$
8 \lambda(\lambda-1)^{2}>\left\|\beta^{e}\right\|_{L^{2}}^{2}\left\|\beta^{l}\right\|_{L^{2}}^{2}\left\|\beta^{f}\right\|_{L^{2}}^{2},
$$

then, using Lemma 1, we see that the $L^{2}$-norm of dual variable $\hat{p}_{v}^{e}$ is bounded as follows,

$$
\left\|\hat{p}_{v}^{e}(t)\right\|_{L^{2}\left(\Omega^{e}\right)} \leq \frac{2 \eta_{1}}{\underline{\beta}^{f} P_{\min }^{f}}<\infty
$$

where $P_{\text {min }}^{f}=\min _{t \geq 0}\left\{P^{f}(t)\right\}$ for all time.

\section{Proof of (4.2) in theorem 3:}

Let $v$ and $\tilde{v}$ be two controls and, $\hat{p}_{v}^{k}$ and $\hat{p}_{\tilde{v}}^{k}(k=e, l, f)$ the corresponding solutions of (7.9). We now denote by $\hat{p}^{k}$ the difference between $p_{v}^{k}$ and $p_{\tilde{v}}^{k}$, and these functions satisfy the following system

$$
\left\{\begin{array}{c}
-\frac{\partial}{\partial t} \hat{p}^{e}(t, a)-g^{e}(E(t), a) \frac{\partial}{\partial a} \hat{p}^{e}(t, a)+\lambda \hat{p}^{e}(t, a)+\left(\beta^{e}(E(t), a)+m^{e}(E(t), a)+\alpha_{2} \tilde{v}_{2}(t, a)\right) \hat{p}^{e}(t, a)= \\
\hat{p}^{l}(t, 0) \beta^{e}(E(t), a)-\alpha_{2}\left(v_{2}(t, a)-\tilde{v}_{2}(t, a)\right) \hat{p}_{v}^{e}(t, a), \\
-\frac{\partial}{\partial t} \hat{p}^{l}(t, a)-g^{l}(E(t), a) \frac{\partial}{\partial a} \hat{p}^{l}(t, a)+\lambda \hat{p}^{l}(t, a)+\left(\beta^{l}(E(t), a)+m_{\tilde{v}}^{l}(E(t), a)+\partial_{x} m_{\tilde{v}}^{l} u_{\tilde{v}}^{l}(t, a)\right) \hat{p}^{l}(t, a) \\
\quad+\left(m_{v}^{l}-m_{\tilde{v}}^{l}\right) \hat{p}_{v}^{l}(t, a)+\left(\partial_{x} m_{v}^{l} u_{v}^{l}-\partial_{x} m_{\tilde{v}}^{l} u_{\tilde{v}}^{l}\right) \hat{p}_{v}^{l}(t, a)=\hat{p}^{f}(t, 0) \beta^{l}(a), \\
-\frac{\partial}{\partial t} \hat{p}^{f}(t, a)-g^{f}(E(t), a) \frac{\partial}{\partial a} \hat{p}^{f}(t, a)+\lambda \hat{p}^{f}(t, a)+m^{f}(E(t), a) \hat{p}^{f}(t, a)=\left(1-\alpha_{1} \tilde{v}_{1}(t, a)\right) \hat{p}^{e}(t, 0) \\
\quad \times\left[\beta_{\tilde{v}}^{f}+\partial_{x} \beta_{\tilde{v}}^{f} u_{\tilde{v}}^{l}\right]-\alpha_{1}\left(v_{1}(t, a)-\tilde{v}_{1}(t, a)\right)\left[\beta_{v}^{f}+\partial_{x} \beta_{v}^{f} u_{v}^{l}\right] \hat{p}_{v}^{e}(t, 0) \\
\quad+\left(1-\alpha_{1} \tilde{v}_{1}(t, a)\right) \hat{p}_{v}^{e}(t, 0)\left[\beta_{v}^{f}-\beta_{\tilde{v}}^{f}+u_{v}^{l} \partial_{x} \beta_{v}^{f}-u_{\tilde{v}}^{l} \partial_{x} \beta_{\tilde{v}}^{f}\right], \\
\hat{p}^{k}(T, a)=0, \quad k=e, l, f, \\
\hat{p}^{k}\left(t, L^{k}\right)=0, \quad k=e, l, f,
\end{array}\right.
$$


where $P^{k}(t)=\int_{0}^{L^{k}} u_{v}^{k}(t, a) d a(k=l, f)$ and $u_{v}^{k}$ is given by (2.3). We multiply now the first three equations above, respectively by $\hat{p}^{e}, \hat{p}^{l}$ and $\hat{p}^{f}$ and we integrate the resulting equations on the corresponding domain $\Omega^{k}$ to get

$$
\left\{\begin{aligned}
& \frac{1}{2} \int_{0}^{T}\left(\hat{p}^{e}\right)^{2}(t, 0) d t+\lambda \int_{\Omega^{e}}\left(\hat{p}^{e}\right)^{2}(t, a) d a d t \\
& \quad \leq \int_{\Omega^{e}}\left|\hat{p}^{l}(t, 0) \beta^{e}(E(t), a) \hat{p}^{e}(t, a)\right| d a d t \\
&+\int_{\Omega^{e}}\left|\left[\tilde{v}_{2}(t)-v_{2}(t)\right] \hat{p}_{v}^{e}(t, a) \hat{p}^{e}(t, a)\right| d a d t, \\
& \frac{1}{2} \int_{0}^{T}\left(\hat{p}^{l}\right)^{2}(t, 0) d t+\lambda \int_{\Omega^{l}}\left(\hat{p}^{l}\right)^{2}(t, a) d a d t \leq \int_{\Omega^{l}}\left|\hat{p}^{f}(t, 0) \beta^{l}(E(t), a) \hat{p}^{l}(t, a) d a d t\right| \\
&+\int_{\Omega^{l}}\left|\left[\left(m_{v}^{l}-m_{\tilde{v}}^{l}+u_{v}^{l} \partial_{x} m_{v}^{l}-u_{\tilde{v}}^{l} \partial_{x} m_{\tilde{v}}^{l}\right)\right] \hat{p}_{v}^{l}(t, a) \hat{p}^{l}(t, a)\right| d a d t, \\
& \frac{1}{2} \int_{0}^{T}\left(\hat{p}^{f}\right)^{2}(t, 0) d t+\lambda \int_{\Omega^{f}}\left(\hat{p}^{f}\right)^{2}(t, a) d a d t \leq \int_{\Omega^{f}}\left|\hat{p}^{e}(t, 0)\left[\beta_{\tilde{v}}^{f}+u_{\tilde{v}}^{f} \partial_{x} \beta_{\tilde{v}}^{f}\right] \hat{p}^{f}(t, a)\right| d a d t \\
&+\int_{\Omega^{f}}\left|\left(\tilde{v}_{1}(t)-v_{1}(t)\right) \hat{p}_{v}^{e}(t, 0)\left[\beta_{v}^{f}+u_{v}^{f} \partial_{x} \beta_{v}^{f}\right] \hat{p}^{f}(t, a)\right| d a d t \\
&+\int_{\Omega^{f}}\left|\hat{p}_{v}^{e}(t, 0)\left[\beta_{v}^{f}-\beta_{\tilde{v}}^{f}+u_{\tilde{v}}^{f} \partial_{x} \beta_{v}^{f} x-u_{\tilde{v}}^{f} \partial_{x} \beta_{\tilde{v}}^{f}\right] \hat{p}^{f}(t, a)\right| d a d t .
\end{aligned}\right.
$$

Applying Young's and Cauchy Schwartz's inequalities to the integrals on the righthand sides of these inequalities we are led to the following relations,

$$
\left\{\begin{aligned}
\int_{\Omega^{e}}\left|\hat{p}^{l}(t, 0) \beta^{e}(E(t), a) \hat{p}^{e}(t, a)\right| d a d t \leq & \frac{\epsilon_{1}}{2}\left\|\beta^{e}\right\|_{L^{2}}^{2} \int_{\Omega^{e}}\left(\hat{p}^{e}\right)^{2}(t, a) d a d t \\
& +\frac{1}{2 \epsilon_{1}} \int_{0}^{T}\left(\hat{p}^{l}\right)^{2}(t, 0) d t, \\
\int_{\Omega^{e}}\left|\left[\tilde{v}_{2}(t, a)-v_{2}(t, a)\right] \hat{p}_{v}^{e}(t, a) \hat{p}^{e}(t, a)\right| & d a d t \leq \frac{\epsilon_{2}}{2}\left\|\hat{p}_{v}^{e}\right\|_{L^{2}}^{2} \int_{\Omega^{e}}\left(\hat{p}^{e}\right)^{2}(t, a) d a d t \\
& +\frac{1}{2 \epsilon_{2}} \int_{\Omega^{e}}\left[\tilde{v}_{2}(t, a)-v_{2}(t, a)\right]^{2} d a d t,
\end{aligned}\right.
$$

for the first inequality in (7.11);

$$
\left\{\begin{aligned}
\int_{\Omega^{l}}\left|\hat{p}^{f}(t, 0) \beta^{l}(E(t), a) \hat{p}^{l}(t, a)\right| d a d t \leq & \frac{\epsilon_{3}}{2}\left\|\beta^{l}\right\|_{L^{2}}^{2} \int_{\Omega^{l}}\left(\hat{p}^{l}\right)^{2}(t, a) d a d t \\
& +\frac{1}{2 \epsilon_{3}} \int_{0}^{T}\left(\hat{p}^{f}\right)^{2}(t, 0) d t \\
\int_{\Omega^{l}} \mid\left[\left(m_{v}^{l}-m_{\tilde{v}}^{l}+u_{v}^{l} \partial_{x} m_{v}^{l}-u_{\tilde{v}}^{l} \partial_{x} m_{\tilde{v}}^{l}\right)\right] & \hat{p}_{v}^{l}(t, a) \hat{p}^{l}(t, a) \mid d a d t \leq \frac{m_{K}\left(D^{l}\right)^{2}}{2 \epsilon_{4}}\|v-\tilde{v}\|_{L^{\infty}}^{2} \\
& +\frac{m_{K} \epsilon_{4}}{2}\left\|\hat{p}_{v}^{l}\right\|_{L^{2}}^{2} \int_{\Omega^{l}}\left(\hat{p}^{l}\right)^{2}(t, a) d a d t,
\end{aligned}\right.
$$


for the second inequality in (7.11);

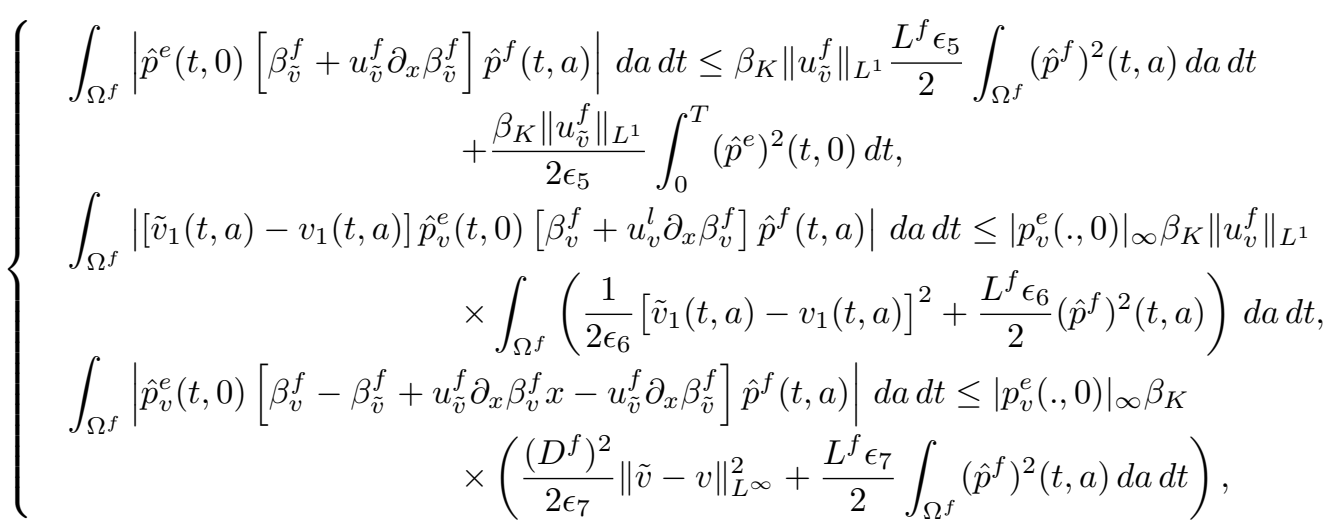

for the last one. Next, we choose $\epsilon_{1}$ and $\epsilon_{2}$ in the inequalities (7.12) as

$$
\epsilon_{1}=\frac{2(\lambda-1)}{\left\|\beta^{e}\right\|_{L^{2}}^{2}}, \quad \epsilon_{2}=\frac{2}{\left\|\hat{p}_{v}^{e}\right\|_{L^{2}}^{2}},
$$

and then the first relation of (7.11) becomes

$$
\frac{1}{2} \int_{0}^{T}\left(\hat{p}^{e}\right)^{2}(t, 0) d t \leq \frac{1}{2 \epsilon_{1}} \int_{0}^{T}\left(\hat{p}^{l}\right)^{2}(t, 0) d t+\frac{1}{2 \epsilon_{2}} \int_{\Omega^{e}}\left[v_{2}(t, a)-\tilde{v}_{2}(t, a)\right]^{2} d a d t .
$$

Similarly, we choose $\epsilon_{3}$ and $\epsilon_{4}$ in the inequalities (7.13) as

$$
\epsilon_{3}=\frac{2(\lambda-1)}{\left\|\beta^{l}\right\|_{L^{2}}^{2}}, \quad \epsilon_{4}=\frac{2}{m_{K}\left\|\hat{p}_{v}^{l}\right\|_{L^{2}}^{2}},
$$

and the second relation of (7.11) becomes

$$
\frac{1}{2} \int_{0}^{T}\left(\hat{p}^{l}\right)^{2}(t, 0) d t \leq \frac{1}{2 \epsilon_{3}} \int_{0}^{T}\left(\hat{p}^{f}\right)^{2}(t, 0) d t+\frac{m_{K}}{\epsilon_{4}}\left(D^{l}\right)^{2}\|v-\tilde{v}\|_{L^{\infty}}^{2} .
$$

Finally, we choose $\epsilon_{5}, \epsilon_{6}$ and $\epsilon_{7}$ in the inequalities of (7.14) as

$$
\epsilon_{5}=\frac{2(\lambda-2)}{\beta_{K} L^{f}\left\|u_{\tilde{v}}^{f}\right\|_{L^{1}}^{2}}, \quad \epsilon_{6}=\frac{2}{L^{f} \beta_{K}\left\|u_{v}^{f}\right\|_{L^{1}}^{2}\left|p_{v}^{e}(., 0)\right|_{\infty}}, \quad \epsilon_{7}=\frac{2}{L^{f} \beta_{K}\left|p_{v}^{e}(., 0)\right|_{\infty}},
$$

and the third relation of (7.11) becomes

$$
\begin{gathered}
\frac{1}{2} \int_{0}^{T}\left(\hat{p}^{f}\right)^{2}(t, 0) d t \leq \frac{\beta_{K}\left|p_{v}^{e}(., 0)\right|_{\infty}\left\|u_{v}^{f}\right\|_{L^{1}}}{2 \epsilon_{6}} \int_{\Omega^{f}}\left[\tilde{v}_{1}(t, a)-v_{1}(t, a)\right]^{2} d a d t \\
\quad+\frac{\beta_{K}\left\|u_{\tilde{v}}^{f}\right\|_{L^{1}}}{2 \epsilon_{5}} \int_{0}^{T}\left(\hat{p}^{e}\right)^{2}(t, 0) d t+\frac{\beta_{K}\left|p_{v}^{e}(., 0)\right|_{\infty}}{2 \epsilon_{5}}\left(D^{f}\right)^{2}\|\tilde{v}-v\|_{L^{\infty}}^{2} .
\end{gathered}
$$


We substitute this last bound (7.17) in the inequality (7.16) to get

$$
\begin{aligned}
\frac{1}{2} \int_{0}^{T}\left(\hat{p}^{l}\right)^{2}(t, 0) d t \leq \frac{1}{\epsilon_{3}}\left[\frac{\beta_{K}\left|p_{v}^{e}(., 0)\right|_{\infty}\left\|u_{v}^{f}\right\|_{L^{1}}}{2 \epsilon_{6}} \int_{\Omega^{f}}\left[\tilde{v}_{1}(t, a)-v_{1}(t, a)\right]^{2} d a d t\right. \\
\left.\quad+\frac{\beta_{K}\left\|u_{\tilde{v}}^{f}\right\|_{L^{1}}}{2 \epsilon_{5}} \int_{0}^{T}\left(\hat{p}^{e}\right)^{2}(t, 0) d t+\frac{\beta_{K}\left|p_{v}^{e}(., 0)\right|_{\infty}}{2 \epsilon_{5}}\left(D^{f}\right)^{2}\|\tilde{v}-v\|_{L^{\infty}}^{2}\right] \\
\quad+\frac{m_{K}}{\epsilon_{4}}\left(D^{l}\right)^{2}\|v-\tilde{v}\|_{L^{\infty}}^{2},
\end{aligned}
$$

and now substitute this inequality into (7.15) to see that

$$
\begin{aligned}
\left(\frac{1}{2}-\right. & \left.\frac{\beta_{K}\left\|u_{\tilde{v}}^{f}\right\|_{L^{1}}}{2 \epsilon_{1} \epsilon_{3} \epsilon_{5}}\right) \int_{0}^{T}\left(\hat{p}^{e}\right)^{2}(t, 0) d t \leq \frac{1}{\epsilon_{1} \epsilon_{3}}\left[\frac{\beta_{K}\left|p_{v}^{e}(., 0)\right|_{\infty}\left\|u_{v}^{f}\right\|_{L^{1}}}{2 \epsilon_{6}}\right. \\
& \left.\times \int_{\Omega^{f}}\left[\tilde{v}_{1}(t, a)-v_{1}(t, a)\right]^{2} d a d t+\frac{\beta_{K}\left|p_{v}^{e}(., 0)\right|_{\infty}}{2 \epsilon_{5}}\left(D^{f}\right)^{2}\|\tilde{v}-v\|_{L^{\infty}}^{2}\right] \\
& +\frac{m_{K}}{\epsilon_{1} \epsilon_{4}}\left(D^{l}\right)^{2}\|v-\tilde{v}\|_{L^{\infty}}^{2}+\frac{1}{2 \epsilon_{2}} \int_{\Omega^{e}}\left[\tilde{v}_{2}(t, a)-v_{2}(t, a)\right]^{2} d a d t .
\end{aligned}
$$

If we take $\lambda$ such that

$$
8(\lambda-2)(\lambda-1)^{2}>\left\|\beta^{e}\right\|_{L^{2}}^{2}\left\|\beta^{l}\right\|_{L^{2}}^{2} \beta_{K} L^{f}\left\|u_{\tilde{v}}^{f}\right\|_{L^{1}},
$$

then inequality (7.18) can be rewritten as

$$
\begin{aligned}
\int_{0}^{T}\left(\hat{p}^{e}\right)^{2}(t, 0) d t \leq \frac{1}{\epsilon_{1} \epsilon_{3}}\left[\frac{\beta_{K}\left|p_{v}^{e}(., 0)\right|_{\infty}\left\|u_{v}^{f}\right\|_{L^{1}}}{2 \epsilon_{6}}\right. \\
\left.\quad \times \int_{\Omega^{f}}\left[\tilde{v}_{1}(t, a)-v_{1}(t, a)\right]^{2} d a d t+\frac{\beta_{K}\left|p_{v}^{e}(., 0)\right|_{\infty}}{2 \epsilon_{5}}\left(D^{f}\right)^{2}\|\tilde{v}-v\|_{L^{\infty}}^{2}\right] \\
\quad+\frac{m_{K}}{\epsilon_{1} \epsilon_{4}}\left(D^{l}\right)^{2}\|v-\tilde{v}\|_{L^{\infty}}^{2}+\frac{1}{2 \epsilon_{2}} \int_{\Omega^{e}}\left[\tilde{v}_{2}(t, a)-v_{2}(t, a)\right]^{2} d a d t .
\end{aligned}
$$

We now let

$$
\begin{aligned}
& G_{1}=\left[\frac{\beta_{K}\left|p_{v}^{e}(., 0)\right|_{\infty}\left\|u_{v}^{f}\right\|_{L^{1}}}{2 \epsilon_{1} \epsilon_{3} \epsilon_{6}}+\frac{\beta_{K}\left|p_{v}^{e}(., 0)\right|_{\infty}}{2 \epsilon_{1} \epsilon_{3} \epsilon_{5}}\left(D_{1}^{f}\right)^{2}+\frac{m_{K}}{\epsilon_{1} \epsilon_{4}}\left(D_{1}^{l}\right)^{2}\right]^{1 / 2}, \\
& G_{2}=\left[\frac{\beta_{K}\left|p_{v}^{e}(., 0)\right|_{\infty}}{2 \epsilon_{1} \epsilon_{3} \epsilon_{5}}\left(D_{2}^{f}\right)^{2}+\frac{m_{K}}{\epsilon_{1} \epsilon_{4}}\left(D_{2}^{l}\right)^{2}+\frac{1}{2 \epsilon_{2}}\right]^{1 / 2},
\end{aligned}
$$

and then (7.18) leads to the first inequality of theorem 3 , that is

$$
\left|p_{v}^{e}(t, 0)-p_{\tilde{v}}^{e}(t, 0)\right| \leq\left|\hat{p}^{e}(t, 0)\right| \leq G \cdot\|v-\tilde{v}\|_{L^{\infty}},
$$

for all $t$, with $G=\max \left\{G_{1}, G_{2}\right\}$.

\section{Proof of (4.3) of theorem (3):}

We consider systems (7.11), (7.12), (7.13) and (7.14), and we choose the constants $\epsilon_{i}$, for $i=1, \ldots, 7$ as in the previous proof except that $\epsilon_{1}$ is given now by

$$
\epsilon_{1}=\frac{2(\lambda-2)}{\left\|\beta^{e}\right\|_{L^{2}}^{2}}
$$


February 16, $2015 \quad$ 10:27

26 Picart, Milner

The same argument just used for the first estimate in theorem 3 now leads to the second estimate in the theorem, with $H=G$ defined in (7.19). 